\title{
RSK Insertion for Set Partitions and Diagram Algebras
}

\author{
Tom Halverson* and Tim Lewandowski* \\ Department of Mathematics and Computer Science \\ Macalester College, Saint Paul, MN 55105 USA \\ halverson@macalester.edu tim.lewandowski@gmail.com
}

Submitted: Jun 30, 2005; Accepted: Nov 2, 2005; Published: Dec 5, 2005

Mathematics Subject Classifications: 05A19, 05E10, 05A18

In honor of Richard Stanley on his 60th birthday.

\begin{abstract}
We give combinatorial proofs of two identities from the representation theory of the partition algebra $\mathbb{C} A_{k}(n), n \geq 2 k$. The first is $n^{k}=\sum_{\lambda} f^{\lambda} m_{k}^{\lambda}$, where the sum is over partitions $\lambda$ of $n, f^{\lambda}$ is the number of standard tableaux of shape $\lambda$, and $m_{k}^{\lambda}$ is the number of "vacillating tableaux" of shape $\lambda$ and length $2 k$. Our proof uses a combination of Robinson-Schensted-Knuth insertion and jeu de taquin. The second identity is $B(2 k)=\sum_{\lambda}\left(m_{k}^{\lambda}\right)^{2}$, where $B(2 k)$ is the number of set partitions of $\{1, \ldots, 2 k\}$. We show that this insertion restricts to work for the diagram algebras which appear as subalgebras of the partition algebra: the Brauer, Temperley-Lieb, planar partition, rook monoid, planar rook monoid, and symmetric group algebras.
\end{abstract}

\section{Introduction}

Two fundamental identities in the representation theory of the symmetric group $S_{k}$ are

$$
\text { (a) } \quad n^{k}=\sum_{\substack{\lambda \vdash k \\ \ell(\lambda) \leq n}} f^{\lambda} d_{\lambda}, \quad \text { and } \quad \text { (b) } k !=\sum_{\lambda \vdash k}\left(f^{\lambda}\right)^{2} \text {, }
$$

where $\lambda$ varies over partitions of the integer $k$ of length $\ell(\lambda) \leq n, f^{\lambda}$ is the number of standard Young tableaux of shape $\lambda$, and $d_{\lambda}$ is the number of column strict tableaux of shape $\lambda$ with entries from $\{1, \ldots, n\}$. The Robinson-Schensted-Knuth (RSK) insertion algorithm provides a a bijection between sequences $\left(i_{1}, \ldots, i_{k}\right), 1 \leq i_{j} \leq n$, and pairs $\left(P_{\lambda}, Q_{\lambda}\right)$ consisting of a standard Young tableau $P_{\lambda}$ of shape $\lambda$ and a column strict tableau

${ }^{*}$ Research supported in part by National Science Foundation Grant DMS0401098. 
$Q_{\lambda}$ of shape $\lambda$, thus providing a combinatorial proof of (1.1.a). If we restrict $i_{1}, \ldots, i_{k}$ to be a permutation of $1, \ldots, k$, then $Q_{\lambda}$ is a standard tableau and we have a proof of (1.1.b).

Identity (1.1.b) comes from the decomposition of the group algebra $\mathbb{C}\left[S_{k}\right]$ into irreducible $S_{k}$-modules $V^{\lambda}, \lambda \vdash k$, where $\operatorname{dim}\left(V^{\lambda}\right)=f^{\lambda}$ and the multiplicity of $V^{\lambda}$ in $\mathbb{C}\left[S_{k}\right]$ is also $f^{\lambda}$. Identity (1.1.a) comes from the Schur-Weyl duality between $S_{k}$ and the general linear group $G L_{n}(\mathbb{C})$ on the $k$-fold tensor product $V^{\otimes k}$ of the fundamental representation $V$ of $G L_{n}(\mathbb{C})$. There is an action of $S_{k}$ on $V^{\otimes k}$ by tensor place permutations, and via this action, $\mathbb{C}\left[S_{k}\right]$ is isomorphic to the centralizer algebra $\operatorname{End}_{G L_{n}(\mathbb{C})}\left(V^{\otimes k}\right)$. As a bimodule for $S_{k} \times G L_{n}(\mathbb{C})$,

$$
V^{\otimes k} \cong \bigoplus_{\lambda \vdash k} S^{\lambda} \otimes V^{\lambda}
$$

where $S^{\lambda}$ is an irreducible $S_{k}$-module of dimension $f^{\lambda}$ and $V^{\lambda}$ is an irreducible $G L_{r}(\mathbb{C})$ module of dimension $d_{\lambda}$. We get (1.1.a) by computing dimensions on each side of (1.2).

Brauer $[\mathrm{Br}]$ defined an algebra $\mathbb{C} B_{k}(n)$, which is isomorphic to the centralizer algebra of the orthogonal group $O_{n}(\mathbb{C}) \subseteq G L_{n}(\mathbb{C})$, when $n \geq 2 k$, i.e., $\mathbb{C} B_{k}(n) \cong \operatorname{End}_{O_{n}(\mathbb{C})}\left(V^{\otimes k}\right)$. The dimension of the Brauer algebra is $(2 k-1) ! !=(2 k-1)(2 k-3) \cdots 3 \cdot 1$. Since $O_{n}(\mathbb{C}) \subseteq G L_{n}(\mathbb{C})$, their centralizers satisfy $\mathbb{C} B_{k}(n) \supseteq \mathbb{C}\left[S_{k}\right]$. Berele $[$ Be] generalized the RSK correspondence to give combinatorial proof of the $\mathbb{C} B_{k}(n)$-analog of (1.1.a). Sundaram [Sun] (see also [Ter]) gave a combinatorial proof of the $\mathbb{C} B_{k}(n)$-analog of (1.1.a).

We now take this restriction further to $S_{n-1} \subseteq S_{n} \subseteq O_{n}(\mathbb{C}) \subseteq G L_{n}(\mathbb{C})$, where $S_{n}$ is viewed as the subgroup of permutation matrices in $G L_{n}(\mathbb{C})$ and $S_{n-1} \subseteq S_{n}$ corresponds to the permutations that fix $n$. Under this restriction, $V$ is the permutation representation of $S_{n}$, and when $n \geq 2 k$, the centralizer algebras are the partition algebras,

$$
\mathbb{C} A_{k}(n) \cong \operatorname{End}_{S_{n}}\left(V^{\otimes k}\right) \text { and } \quad \mathbb{C} A_{k+\frac{1}{2}}(n) \cong \operatorname{End}_{S_{n-1}}\left(V^{\otimes k}\right) .
$$

The partition algebra $\mathbb{C} A_{k}(n)$ first appeared independently in the work of Martin [Mar1, Mar2, Mar3] and Jones [Jo] arising from applications in statistical mechanics. See [HR2] for a survey paper on partition algebras.

For $k \in \mathbb{Z}_{>0}$ and $n \geq 2 k$, the $\mathbb{C}$-algebras $\mathbb{C} A_{k}(n)$ and $\mathbb{C} A_{k+\frac{1}{2}}(n)$ are semisimple with bases indexed by set partitions of $\{1, \ldots, 2 k\}$ and $\{1, \ldots, 2 k+1\}$, respectively. Thus, $\left.\operatorname{dim}\left(\mathbb{C} A_{k}(n)\right)\right)=B(2 k)$ and $\left.\operatorname{dim}\left(\mathbb{C} A_{k+\frac{1}{2}}(n)\right)\right)=B(2 k+1)$, where $B(\ell)$ is the $\ell$ th Bell number. Define,

$$
\Lambda_{n}^{k}=\left\{\lambda \vdash n|| \lambda \mid-\lambda_{1} \leq k\right\},
$$

(these are partitions of $n$ with at most $k$ boxes below the first row of their Young diagram). Then the irreducible representations of $\mathbb{C} A_{k}(n)$ are indexed by partitions in the set $\Lambda_{n}^{k}$, and the irreducible representations of $\mathbb{C} A_{k+\frac{1}{2}}(n)$ are indexed by partitions in the set $\Lambda_{n-1}^{k}$.

Using the Schur-Weyl duality between $S_{n}$ and $\mathbb{C} A_{k}(n)$ we get the identity

$$
n^{k}=\sum_{\lambda \in \Lambda_{n}^{k}} f^{\lambda} m_{k}^{\lambda}
$$


where $m_{k}^{\lambda}$ is the number of vacillating tableaux of shape $\lambda$ and length $2 k$ (defined in Section 2.2), which are sequences of integer partitions in the Bratteli diagram of $\mathbb{C} A_{k}(n)$. Identity (1.4) is the partition algebra analog of (1.1.a). In Section 3, we prove (1.4) using RSK column insertion and jeu de taquin. Decomposing $\mathbb{C} A_{k}(n)$ as a bimodule for $\mathbb{C} A_{k}(n) \otimes \mathbb{C} A_{k}(n)$ gives

$$
B(2 k)=\sum_{\lambda}\left(m_{k}^{\lambda}\right)^{2}
$$

which is the $\mathbb{C} A_{k}(n)$-analog of (1.1.b). In Section 4, we give a bijective proof of (1.5) that contains as a special cases the RSK algorithms for $\mathbb{C} S_{k}$ and $\mathbb{C} B_{k}(n)$.

Martin and Rollet $[\mathrm{MR}]$ have given a different combinatorial proof of the second identity (1.5). Their bijection has the elegant property that pairs of paths in the difference between the Bratteli diagrams of $\mathbb{C} A_{k}(\ell)$ and $\mathbb{C} A_{k}(\ell-1)$ are in exact correspondence with the set partitions of $\{1, \ldots, 2 k\}$ into $\ell$ parts. The advantages of the correspondence in this paper are:

1. Our algorithm for (1.5) contains, as special cases, the known RSK correspondences for a number of diagram algebras which appear as subalgebras of $\mathbb{C} A_{k}(n)$ :

(a) The group algebra of the symmetric group $\mathbb{C} S_{k}$,

(b) The Brauer algebra $\mathbb{C} B_{k}(n)$,

(c) The Temperley-Lieb algebra $\mathbb{C} T_{k}(n)$,

(d) The planar partition algebra $\mathbb{C} P_{k}(n)$.

(e) The rook monoid algebra $\mathbb{C} R_{n}$ and the planar rook monoid algebra $\mathbb{C} P R_{n}$.

Thus we obtain combinatorial proofs of the analog of (1.5) for each of these algebras (see equations (5.1), (5.2), (5.3), and (5.4)).

2. Using Fomin growth diagrams we show that our algorithm for (1.5) is symmetric in the sense that if $d \rightarrow(P, Q)$ then $\operatorname{flip}(d) \mapsto(Q, P)$ where flip $(d)$ is the diagram $d$ flipped over its horizontal axis. This is the generalization of the property for the symmetric group that if $\pi \rightarrow(P, Q)$ then $\pi^{-1} \rightarrow(Q, P)$. As a consequence, we show that the number of symmetric diagrams equals the sum of the dimensions of the irreducible representations (for each of the diagram algebras mentioned in item 1 above).

3. Our algorithms for the bijections in (1.4) and (1.5) each use iterations of RSK insertion and jeu de taquin (see equations (3.4) and (4.4)).

The main idea for the algorithm in this paper came from a bijection of R. Stanley between fixed point free involutions in the symmetric group $S_{2 k}$ and Brauer diagrams. This led to the insertion scheme of Sundaram in [Sun] for the Brauer algebra. After we distributed a preliminary version of this paper, R. Stanley and colleagues independently came out with the paper [CDDSY], which studies the crossing and nesting properties of an extended version of the insertion used in this paper for the bijection in (1.5). We have adopted the term "vacillating tableaux" from [CDDSY], and we use the crossing property 
of [CDDSY] to show that our algorithm restricts appropriately to the planar partition algebra.

In Section 2, we give two equivalent notations for vacillating tableaux. We show that when $n \geq 2 k$, there is a bijection between $\Lambda_{n}^{k}$ and $\Gamma_{k}=\{\lambda \vdash t \mid 0 \leq t \leq k\}$ given by removing the first part of $\lambda \in \Lambda_{n}^{k}$ to produce $\lambda^{*} \in \Gamma_{k}$. We use two notations because the $\Lambda_{n}^{k}$ notation is best suited to state and prove (1.4) and the $\Gamma_{k}$ notation is best suited to state and prove (1.5). The vacillating tableaux in [CDDSY] are the same as those in this paper under the $\Gamma_{k}$-notation, however we use the term "vacillating tableaux" for both notations.

\section{The Partition Algebra and Vacillating Tableaux}

For $k \in \mathbb{Z}_{>0}$, let

$$
\begin{aligned}
A_{k} & =\left\{\text { set partitions of }\left\{1,2, \ldots, k, 1^{\prime}, 2^{\prime}, \ldots, k^{\prime}\right\}\right\}, \quad \text { and } \\
A_{k+\frac{1}{2}} & =\left\{d \in \Pi_{k+1} \mid(k+1) \text { and }(k+1)^{\prime} \text { are in the same block }\right\} .
\end{aligned}
$$

The propagating number of $d \in A_{k}$ is

$$
\operatorname{pn}(d)=\left(\begin{array}{l}
\text { the number of blocks in } d \text { that contain both an element } \\
\text { of }\{1,2, \ldots, k\} \text { and an element of }\left\{1^{\prime}, 2^{\prime}, \ldots, k^{\prime}\right\}
\end{array}\right) .
$$

For convenience, represent a set partition $d \in A_{k}$ by a graph with $k$ vertices in the top row, labeled $1, \ldots, k$, and $k$ vertices in the bottom row, labeled $1^{\prime}, \ldots, k^{\prime}$, with vertex $i$ and vertex $j$ connected by a path if $i$ and $j$ are in the same block of the set partition $d$. For example,

$\begin{array}{lllllllllll}1 & 2 & 3 & 4 & 5 & 6 & 7 & 8\end{array}$

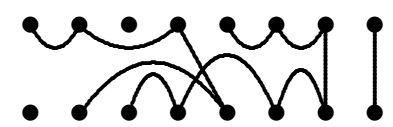

represents $\left\{\left\{1,2,4,2^{\prime}, 5^{\prime}\right\},\{3\},\left\{5,6,7,3^{\prime}, 4^{\prime}, 6^{\prime}, 7^{\prime}\right\},\left\{8,8^{\prime}\right\},\left\{1^{\prime}\right\}\right\}$,

$\begin{array}{llllllllllll}1^{\prime} & 2^{\prime} & 3^{\prime} & 4^{\prime} & 5^{\prime} & 6^{\prime} & 7^{\prime} & 8^{\prime}\end{array}$

and has propagating number 3. The graph representing $d$ is not unique.

We compose two partition diagrams $d_{1}$ and $d_{2}$ as follows. Place $d_{1}$ above $d_{2}$ and identify each vertex $j^{\prime}$ in the bottom row of $d_{1}$ with the corresponding vertex $j$ in the top row of $d_{2}$. This new super diagram is a graph $g$ on 3 rows of vertices. Remove any connected components of $g$ that live entirely in the middle row. Then make a partition diagram $d_{3} \in A_{k}$ so that two vertices in $d_{3} \in A_{k}$ are in the same connected component if and only if the corresponding vertices in the top or bottom rows of $g$ are in the same connected components. Then let $d_{1} \circ d_{2}=d_{3}$. For example, if

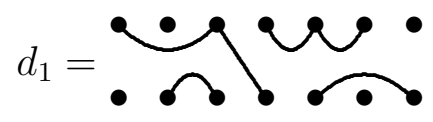

and

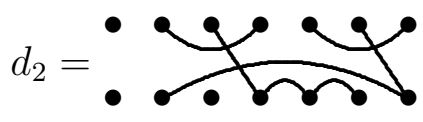


then

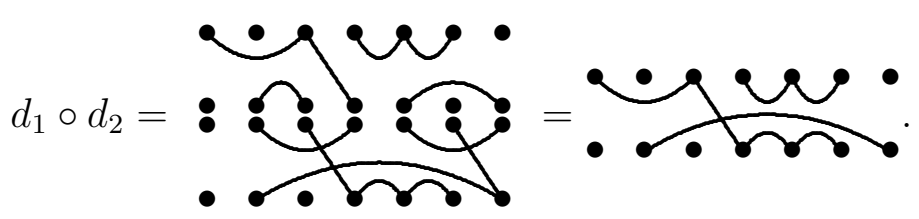

Diagram multiplication makes $A_{k}$ into an associative monoid with identity, $1=\mathbf{q} \boldsymbol{?} \cdots \boldsymbol{?}$, and the propagating number satisfies $\operatorname{pn}\left(d_{1} \circ d_{2}\right) \leq \min \left(\operatorname{pn}\left(d_{1}\right), \operatorname{pn}\left(d_{2}\right)\right)$.

For $k \in \frac{1}{2} \mathbb{Z}_{>0}$ and $n \in \mathbb{C}$, the partition algebra $\mathbb{C} A_{k}(n)=\mathbb{C}$ span- $\left\{d \in A_{k}\right\}$ is an associative algebra over $\mathbb{C}$ with basis $A_{k}$. Multiplication in $\mathbb{C} A_{k}(n)$ is defined by

$$
d_{1} d_{2}=n^{\ell}\left(d_{1} \circ d_{2}\right)
$$

where $\ell$ is the number of blocks removed from the the middle row when constructing the composition $d_{1} \circ d_{2}$. In the example above $d_{1} d_{2}=n^{2} d_{1} \circ d_{2}$.

For each $k \in \mathbb{Z}_{>0}$, the following are submonoids of the partition monoid $A_{k}$ :

$$
\begin{aligned}
& S_{k}=\left\{d \in A_{k} \mid \operatorname{pn}(d)=k\right\}, \quad I_{t}=\left\{d \in A_{k} \mid \operatorname{pn}(d) \leq t\right\}, \quad 0<t \leq k, \\
& B_{k}=\left\{d \in A_{k} \mid \begin{array}{l}
\text { all blocks of } d \text { have size } 2\}, \\
R_{k}=\left\{d \in A_{k} \mid \begin{array}{l}
\text { all blocks of } d \text { have at most one vertex in }\{1, \ldots k\} \\
\text { and at most one vertex in }\left\{1^{\prime}, \ldots k^{\prime}\right\}
\end{array}\right.
\end{array}\right\} .
\end{aligned}
$$

Here, $S_{k}$ is the symmetric group, $B_{k}$ is the Brauer monoid, and $R_{k}$ is the rook monoid. A set partition is planar [Jo] if it can be represented as a graph without edge crossings inside of the rectangle formed by its vertices. The following are planar submonoids,

$$
P_{k}=\left\{d \in A_{k} \mid d \text { is planar }\right\}, \quad T_{k}=B_{k} \cap P_{k}, \quad P R_{k}=R_{k} \cap P_{k}, \quad 1=S_{k} \cap P_{k} .
$$

Here, $T_{k}$ is the Temperley-Lieb monoid. Examples of diagrams in the various submonoids are:

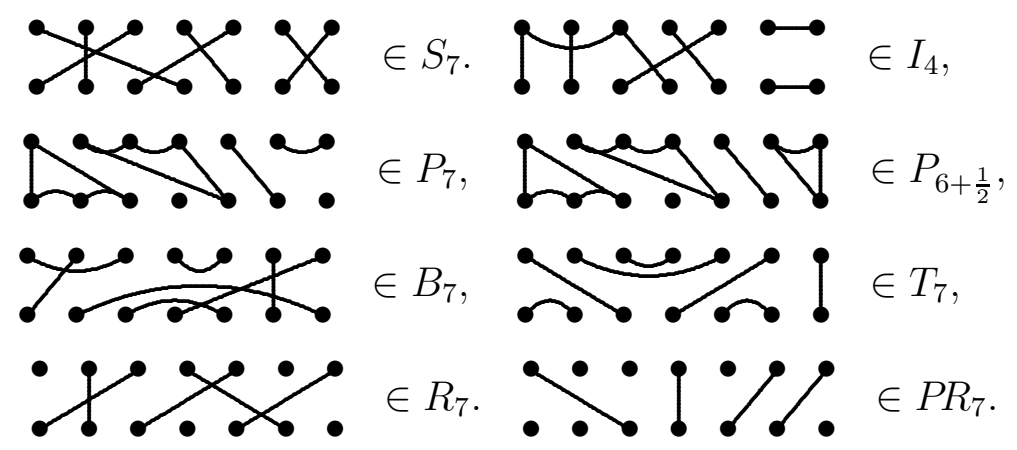

For each monoid, we make an associative algebra in the same way that we construct the partition algebra $\mathbb{C} A_{k}(n)$ from the partition monoid $A_{k}(n)$. For example, we obtain the the Brauer algebra $\mathbb{C} B_{k}(n)$, the Temperley-Lieb algebra $\mathbb{C} T_{k}(n)$, the group algebra of the symmetric group $\mathbb{C} S_{k}$ in this way. Multiplication in the rook monoid algebra $\mathbb{C} R_{k}$ is done without the coefficient $n^{\ell}$ (see [Ha]). 
For $\ell \in \mathbb{Z}_{>0}$, the Bell number $B(\ell)$ is the number of set partitions of $\{1,2, \ldots, \ell\}$, the Catalan number is $C(\ell)=\frac{1}{\ell+1}\left(\begin{array}{c}2 \ell \\ \ell\end{array}\right)=\left(\begin{array}{c}2 \ell \\ \ell\end{array}\right)-\left(\begin{array}{c}2 \ell \\ \ell+1\end{array}\right)$, and $(2 \ell) ! !=(2 \ell-1) \cdot(2 \ell-3) \cdots 5 \cdot 3 \cdot 1$. These have generating functions (see [Sta, 1.24f, and 6.2]),

$$
\sum_{\ell \geq 0} B(\ell) \frac{z^{\ell}}{\ell !}=\exp \left(e^{z}-1\right), \quad \sum_{\ell \geq 0} C(\ell-1) z^{\ell}=\frac{1-\sqrt{1-4 z}}{2 z},
$$

and

$$
\sum_{\ell \geq 0}(2(\ell-1)) ! ! \frac{z^{\ell}}{\ell !}=\frac{1-\sqrt{1-2 z}}{z} .
$$

For $k \in \frac{1}{2} \mathbb{Z}_{>0}$, the monoids have cardinality

$$
\operatorname{Card}\left(A_{k}\right)=B(2 k), \quad \operatorname{Card}\left(P_{k}\right)=\operatorname{Card}\left(T_{2 k}\right)=C(2 k), \quad \text { for } k \in \frac{1}{2} \mathbb{Z}_{>0}
$$

and

$$
\begin{aligned}
& \operatorname{Card}\left(S_{k}\right)=k !, \quad \operatorname{Card}\left(B_{k}\right)=(2 k) ! !, \\
& \operatorname{Card}\left(R_{k}\right)=\sum_{\ell=0}^{k}\left(\begin{array}{l}
k \\
\ell
\end{array}\right)^{2} \ell ! \quad \operatorname{Card}\left(P R_{k}\right)=\left(\begin{array}{c}
2 k \\
k
\end{array}\right), \quad \text { for } k \in \mathbb{Z}_{>0} .
\end{aligned}
$$

\subsection{Schur-Weyl Duality Between $S_{n}$ and $\mathbb{C} A_{k}(n)$}

The irreducible representations of $S_{n}$ are indexed by integer partitions of $n$. If $\lambda=$ $\left(\lambda_{1}, \ldots, \lambda_{\ell}\right) \in\left(\mathbb{Z}_{\geq 0}\right)^{\ell}$ with $\lambda_{1} \geq \cdots \geq \lambda_{\ell}$ and $\lambda_{1}+\cdots+\lambda_{\ell}=n$, then $\lambda$ is a partition of $n$, denoted $\lambda \vdash n$. If $\lambda \vdash n$, then we write $|\lambda|=n$. If $\lambda=\left(\lambda_{1}, \cdots, \lambda_{\ell}\right)$ and $\mu=\left(\mu_{1}, \ldots, \mu_{\ell}\right)$ are partitions such that $\mu_{i} \leq \lambda_{i}$ for each $i$, then we say that $\mu \subseteq \lambda$, and $\lambda / \mu$ is the skew shape given by deleting the boxes of $\mu$ from the Young diagram of $\lambda$.

Let $V$ be the $n$-dimensional permutation representation of the symmetric group $S_{n}$. If we view $S_{n-1} \subseteq S_{n}$ as the subgroup of permutations that fix $n$, then $V$ is isomorphic to the left coset representation $\mathbb{C}\left[S_{n} / S_{n-1}\right]$. Let $V^{\otimes k}$ be the $k$-fold tensor product representation of $V$, and let $V^{\otimes 0}=\mathbb{C}$. From the "tensor identity" (see for example [HR2]), we have the following restriction-induction rule rule for $i \geq 0$,

$$
V^{\otimes(i+1)} \cong V^{\otimes i} \otimes \mathbb{C}\left[S_{n} / S_{n-1}\right] \cong \operatorname{Ind}_{S_{n-1} S_{n}}\left(\operatorname{Res}_{S_{n-1}}^{S_{n}}\left(V^{\otimes i}\right)\right) .
$$

Thus, $V^{\otimes k}$ is obtained from $k$ iterations of restricting to $S_{n-1}$ and inducing back to $S_{n}$.

Let $V^{\lambda}$ denote the irreducible representations of $S_{n}$ indexed by $\lambda \vdash n$. The restriction and induction rules for $S_{n-1} \subseteq S_{n}$ are given by

$$
\begin{aligned}
& \operatorname{Res}_{S_{n-1}}^{S_{n}}\left(V^{\lambda}\right) \cong \bigoplus_{\mu \vdash(n-1), \mu \subseteq \lambda} V^{\mu}, \quad \text { for } \lambda \vdash n \\
& \operatorname{Ind}_{S_{n-1}}^{S_{n}}\left(V^{\mu}\right) \cong \bigoplus_{\lambda \vdash n, \mu \subseteq \lambda,} V^{\lambda}, \quad \text { for } \mu \vdash(n-1) .
\end{aligned}
$$


In each case $\lambda / \mu$ consists of a single box. Starting with the trivial representation $V^{(n)} \cong \mathbb{C}$ and iterating the restriction (2.4) and induction (2.5) rules, we see that the irreducible $S_{n}$-representations that appear in $V^{\otimes k}$ are labeled by the partitions in

$$
\Lambda_{n}^{k}=\left\{\lambda \vdash n|| \lambda \mid-\lambda_{1} \leq k\right\}
$$

and the irreducible $S_{n-1}$-representations that appear in $V^{\otimes k}$ are labeled by the partitions in $\Lambda_{n-1}^{k}$.

There is an action of $\mathbb{C} A_{k}(n)$ on $V^{\otimes k}$ (see [Jo, MR, HR2]) that commutes with $S_{n}$ and maps $\mathbb{C} A_{k}(n)$ surjectively onto the centralizer $\operatorname{End}_{S_{n}}\left(V^{\otimes k}\right)$. This generalizes the actions of $\mathbb{C}\left[S_{k}\right]$, by place permutations, and $B_{k}(n)$ on $V^{\otimes k}$. Furthermore, when $n \geq 2 k$ we have

$$
\mathbb{C} A_{k}(n) \cong \operatorname{End}_{S_{n}}\left(V^{\otimes k}\right) \quad \text { and } \quad \mathbb{C} A_{k+\frac{1}{2}}(n) \cong \operatorname{End}_{S_{n-1}}\left(V^{\otimes k}\right) .
$$

By convention, we let $\mathbb{C} A_{0}(n)=\mathbb{C} A_{\frac{1}{2}}(n)=\mathbb{C}$. Since anything that commutes with $S_{n}$ on $V^{\otimes k}$ will also commute with $S_{n-1}$, we have $\mathbb{C} A_{k}(n) \subseteq \mathbb{C} A_{k+\frac{1}{2}}(n)$, and thus

$$
\mathbb{C} A_{0}(n) \subseteq \mathbb{C} A_{\frac{1}{2}}(n) \subseteq \mathbb{C} A_{1}(n) \subseteq \mathbb{C} A_{1 \frac{1}{2}}(n) \subseteq \cdots \subseteq \mathbb{C} A_{\left(k-\frac{1}{2}\right.}(n) \subseteq \mathbb{C} A_{k}(n) .
$$

The Bratteli diagram for $\mathbb{C} A_{k}(n)$ consists of rows of vertices, with the rows labeled by $0, \frac{1}{2}, 1,1 \frac{1}{2}, \ldots, k$, such that the vertices in row $i$ are $\Lambda_{n}^{i}$ and the vertices in row $i+\frac{1}{2}$ are $\Lambda_{n-1}^{i}$. Two vertices are connected by an edge if they are in consecutive rows and they differ by exactly one box. Figure 1 shows the Bratteli diagram for $\mathbb{C} A_{k}(6)$. Let $n \geq 2 k$. By double centralizer theory (see, for example, [HR2]), we know that

(1) The irreducible representations of $\mathbb{C} A_{k}(n)$ can be indexed by $\Lambda_{n}^{k}$, so we let $M_{k}^{\lambda}$ denote the irreducible $\mathbb{C} A_{k}(n)$ representation indexed by $\lambda \in \Lambda_{n}^{k}$.

(2) The decomposition of $V^{\otimes k}$ as an $S_{n} \times \mathbb{C} A_{k}(n)$-bimodule is given by

$$
V^{\otimes k} \cong \bigoplus_{\lambda \in \Lambda_{n}^{k}} V^{\lambda} \otimes M_{k}^{\lambda}
$$

(3) The dimension of $M_{k}^{\lambda}$ equals the multiplicity of $V^{\lambda}$ in $V^{\otimes k}$. The edges in the Bratteli diagram exactly follow the restriction and induction rules in (2.4), and (2.5). and so

$$
m_{k}^{\lambda}=\operatorname{dim}\left(M_{k}^{\lambda}\right)=\left\{\begin{array}{l}
\text { the number of paths from the top } \\
\text { of the Bratteli diagram to } \lambda
\end{array}\right\} .
$$

\section{$2.2 \quad$ Vacillating Tableaux}

The dimension of the irreducible $S_{n}$-module $V^{\lambda}$ equals the number $f^{\lambda}$ of standard tableaux of shape $\lambda$. A standard tableau of shape $\lambda$ is a filling of the Young diagram of $\lambda$ with the numbers $1,2, \ldots, n$ in such a way that each number appears exactly once, the rows 
Figure 1: Bratteli Diagram for $\mathbb{C} A_{k}(6)$

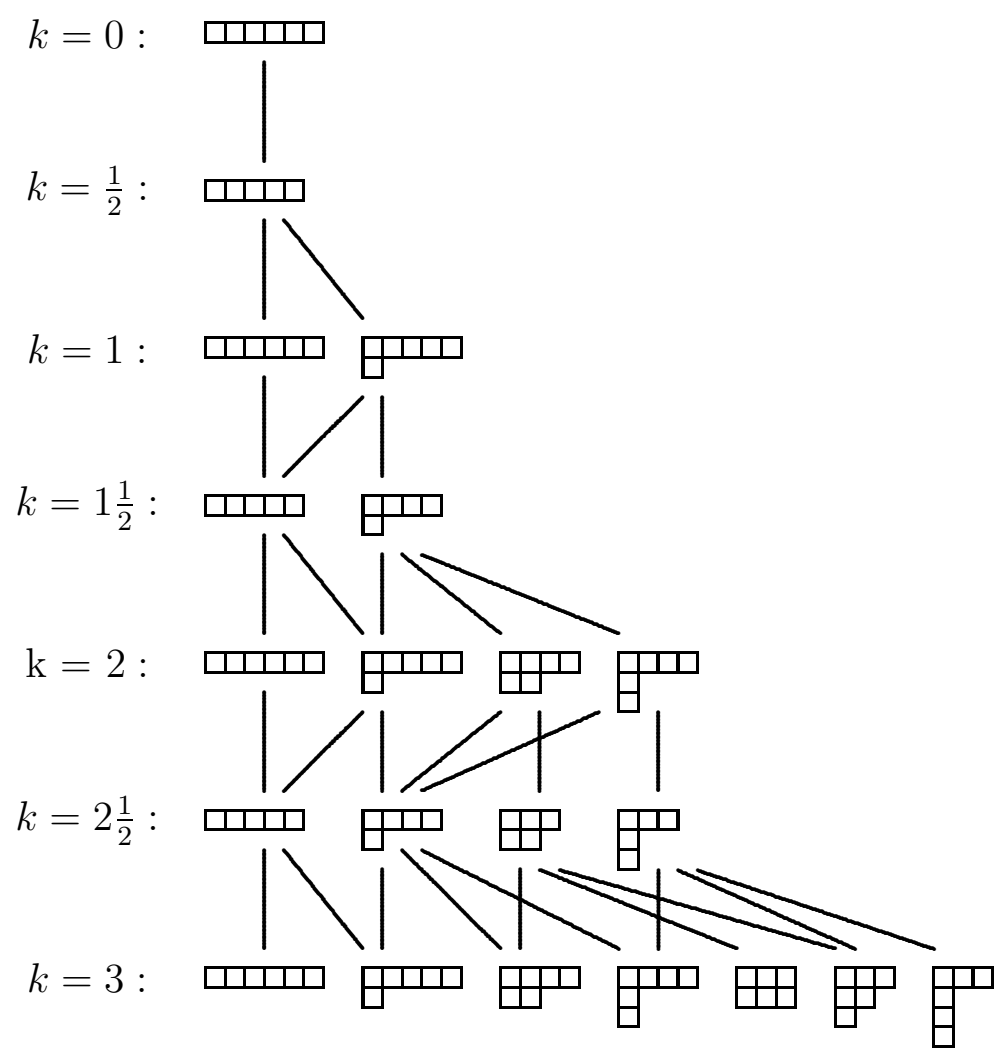

increase from left to right, and the columns increase from top to bottom. We can identify a standard tableaux $T_{\lambda}$ of shape $\lambda$ with a sequence $\left(\emptyset=\lambda^{(0)}, \lambda^{(1)}, \ldots, \lambda^{(n)}=\lambda\right)$ such that $\left|\lambda^{(i)}\right|=i, \lambda^{(i)} \subseteq \lambda^{(i+1)}$, and such that $\lambda^{(i)} / \lambda^{(i-1)}$ is the box containing $i$ in $T_{\lambda}$. For example,

$$
\frac{1: 34}{2 \frac{3}{5}}=(\emptyset, \square, \boxminus, \boxminus, \boxminus, \boxminus)
$$

The sequence $\left(\emptyset=\lambda^{(0)}, \lambda^{(1)}, \ldots, \lambda^{(n)}=\lambda\right)$ is a path in Young's lattice, which is the Bratteli diagram for $S_{n}$. The number of standard tableaux $f^{\lambda}$ can be computed using the hook formula (see $[\mathrm{Sag}, \S 3.10]$ ). We let $\mathcal{S} \mathcal{Y} \mathcal{T}(\lambda)$ denote the set of standard tableaux of shape $\lambda$.

Let $\lambda \in \Lambda_{n}^{k}$. A vacillating tableaux of shape $\lambda$ and length $2 k$ is a sequence of partitions,

$$
\left((n)=\lambda^{(0)}, \lambda^{\left(\frac{1}{2}\right)}, \lambda^{(1)}, \lambda^{\left(1 \frac{1}{2}\right)}, \ldots, \lambda^{\left(k-\frac{1}{2}\right)}, \lambda^{(k)}=\lambda\right),
$$

satisfying, for each $i$,

(1) $\lambda^{(i)} \in \Lambda_{n}^{i}$ and $\lambda^{\left(i+\frac{1}{2}\right)} \in \Lambda_{n-1}^{i}$,

(2) $\lambda^{(i)} \supseteq \lambda^{\left(i+\frac{1}{2}\right)}$ and $\left|\lambda^{(i)} / \lambda^{\left(i+\frac{1}{2}\right)}\right|=1$, 
(3) $\lambda^{\left(i+\frac{1}{2}\right)} \subseteq \lambda^{(i+1)}$ and $\left|\lambda^{(i+1)} / \lambda^{\left(i+\frac{1}{2}\right)}\right|=1$.

The vacillating tableaux of shape $\lambda$ correspond exactly with paths from the top of the Bratteli diagram to $\lambda$. Thus, if we let $\mathcal{V} \mathcal{T}_{k}(\lambda)$ denote the set of vacillating tableaux of shape $\lambda$ and length $k$, then

$$
m_{k}^{\lambda}=\operatorname{dim}\left(M_{k}^{\lambda}\right)=\left|\mathcal{V} \mathcal{T}_{k}(\lambda)\right|
$$

Let $n \geq 2 k$, and for a partition $\lambda$, define $\lambda^{*}$ and $\bar{\lambda}$ as follows,

$$
\begin{aligned}
& \text { if } \quad \lambda=\left(\lambda_{1}, \ldots, \lambda_{\ell}\right) \vdash n, \quad \text { then } \quad \lambda^{*}=\left(\lambda_{2}, \ldots, \lambda_{\ell}\right) \vdash\left(n-\lambda_{1}\right), \\
& \text { if } \quad \lambda=\left(\lambda_{1}, \ldots, \lambda_{\ell}\right) \vdash s \leq k, \text { then } \bar{\lambda}=\left(n-s, \lambda_{1}, \ldots, \lambda_{\ell}\right) \vdash n .
\end{aligned}
$$

Since $n \geq 2 k$ we are guaranteed that $\bar{\lambda}$ is a partition and that $0 \leq\left|\lambda^{*}\right| \leq k$. The sets

$$
\Lambda_{n}^{k}=\left\{\lambda \vdash n|| \lambda^{*} \mid \leq k\right\} \quad \text { and } \quad \Gamma_{k}=\{\lambda \vdash t \mid 0 \leq t \leq k\}
$$

are in bijection with one another using the maps,

$$
\begin{aligned}
\Lambda_{n}^{k} & \rightarrow \Gamma_{k} \\
\lambda & \mapsto \lambda^{*}
\end{aligned} \quad \text { and } \quad \begin{aligned}
\Gamma_{k} & \rightarrow \Lambda_{n}^{k} \\
\lambda & \mapsto \bar{\lambda}
\end{aligned}
$$

Via these bijections, we can use either $\Gamma_{k}$ or $\Lambda_{n}^{k}$ to index the irreducible representations of $\mathbb{C} A_{k}(n)$. For example, the following sequences represent the same vacillating tableau $P_{\lambda}$, the first using diagrams from $\Lambda_{n}^{k}$ and the second from $\Gamma_{k}$,

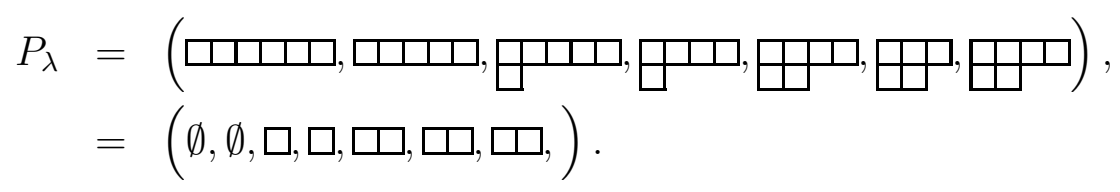

For our bijection in Section 3 we will use $\Lambda_{n}^{k}$, and for our bijection in Section 4 we will use $\Gamma_{k}$. The Bratteli diagram for $\mathbb{C} A_{k}(n), n \geq 2 k$, is given in Figure 2, using labels from $\Gamma_{k}$, along with the number of vacillating tableaux for each shape $\lambda$.

\section{A Bijective Proof of $n^{k}=\sum_{\lambda \in \Lambda_{n}^{k}} f^{\lambda} m_{k}^{\lambda}$}

Comparing dimensions on both sides of the identity (2.9) gives

$$
n^{k}=\sum_{\lambda \in \Lambda_{n}^{k}} f^{\lambda} m_{k}^{\lambda}
$$

For example, when $n=6$ and $k=3$, the $f^{\lambda}$ in the bottom row of Figure 1 (see also Figure 2) are $1,5,9,10,5,16,10$, the corresponding $m_{3}^{\lambda}$ are $5,10,6,6,1,2,1$, and we have $6^{3}=216=1 \cdot 5+5 \cdot 10+9 \cdot 6+10 \cdot 6+5 \cdot 1+16 \cdot 2+10 \cdot 1$. 
Figure 2: Bratteli Diagram for $\mathbb{C} A_{k}(n), n \geq 2 k$.
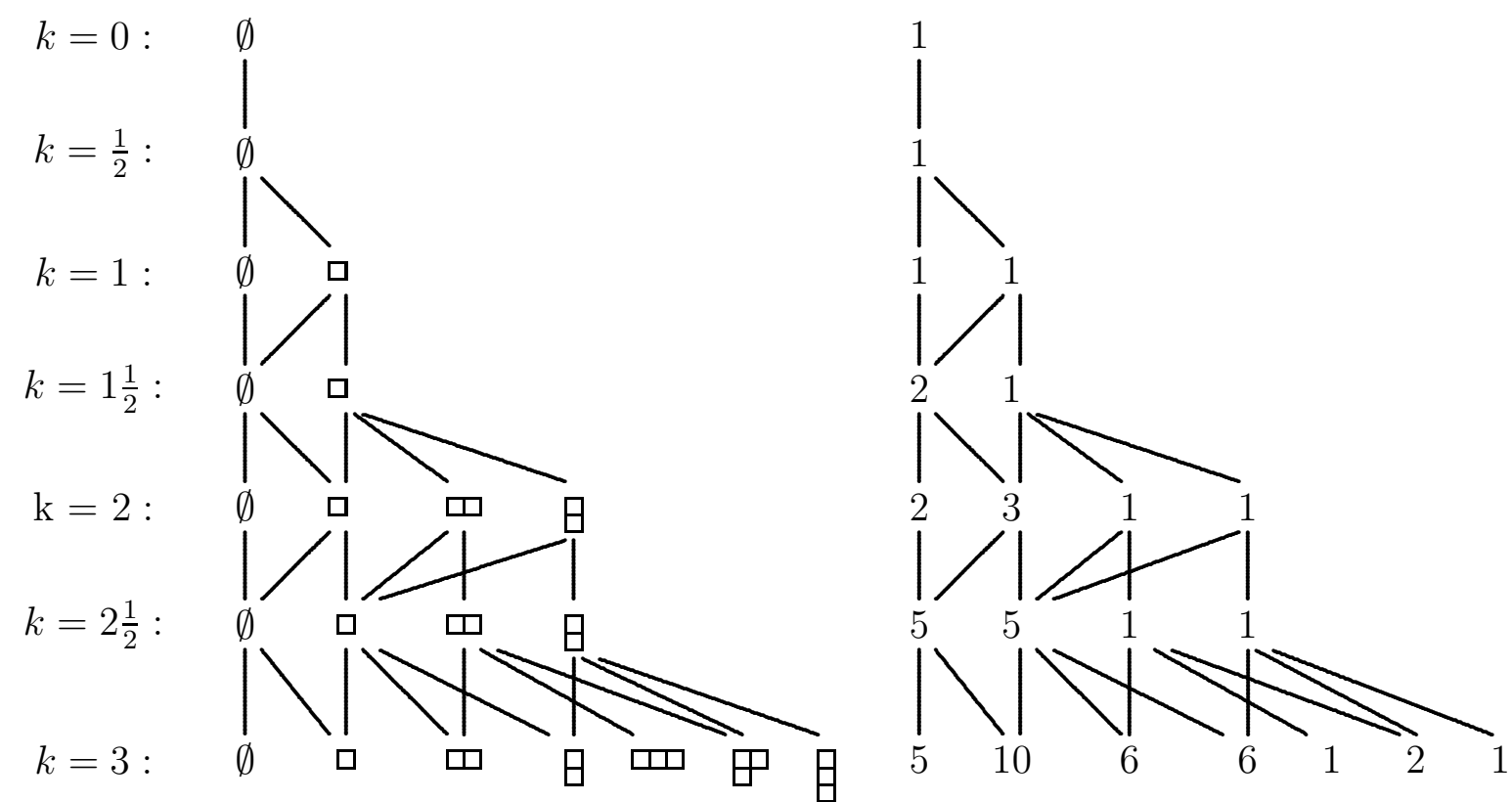

To give a combinatorial proof of (3.1), we need to find a bijection of the form

$$
\left\{\left(i_{1}, \ldots, i_{k}\right) \mid 1 \leq i_{j} \leq n\right\} \longleftrightarrow \bigsqcup_{\lambda \in \Lambda_{n}^{k}} \mathcal{S} \mathcal{Y} \mathcal{T}(\lambda) \times \mathcal{V} \mathcal{T}_{k}(\lambda)
$$

To do so, we construct an invertible function that turns a sequence $\left(i_{1}, \ldots, i_{k}\right)$ of numbers in the range $1 \leq i_{j} \leq n$ into a pair $\left(T_{\lambda}, P_{\lambda}\right)$ consisting of a standard tableaux $T_{\lambda}$ of shape $\lambda$ and vacillating tableaux $P_{\lambda}$ of shape $\lambda$ and length $2 k$ for some $\lambda \in \Lambda_{n}^{k}$. Our bijection uses jeu de taquin and RSK column insertion.

If $T$ is a standard tableau of shape $\lambda \vdash n$, then Schützenberger's [Scü] jeu de taquin provides an algorithm for removing the box containing $x$ from $T$ and producing a standard tableau $S$ of shape $\mu \vdash(n-1)$ with $\mu \subseteq \lambda$ and entries $\{1, \ldots, n\} \backslash\{x\}$. We only need a special case of jeu de taquin for our purposes. See [Sag, §3.7] or [Sta, §A1.2] for the full-strength version and its applications.

If $S$ is a standard tableau, let $S_{i, j}$ denote the entry of $S$ in row $i$ (numbered left-toright) and column $j$ (numbered top-to-bottom). We say that a corner of $S$ is a box whose removal leaves the Young diagram of a partition. Thus the corners of $S$ are the boxes that are both at the end of a row and the end of a column. The following algorithm will delete $x$ from $T$ leaving a standard tableau $S$ with $x$ removed. We denote this process by $x \stackrel{\text { jdt }}{\longleftarrow} T$.

1. Let $c=S_{i, j}$ be the box containing $x$.

2. While $c$ is not a corner, do 
A. Let $c^{\prime}$ be the box containing $\min \left\{S_{i+1, j}, S_{i, j+1}\right\}$;

B. Exchange the positions of $c$ and $c^{\prime}$.

\section{Delete $c$.}

If only one of $S_{i+1, j}, S_{i, j+1}$ exists at step 2.A, then the minimum is taken to be that single value. Below is an example of $2 \stackrel{\text { jdt }}{\longleftarrow} T$,

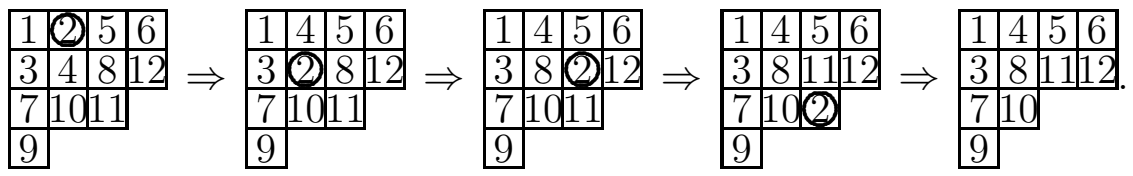

The jeu de taquin algorithm is invertible in the following sense. Suppose that $T$ is a tableaux that is standard but missing an element $x$, and suppose that we are given the position on the border of $T$ from which $x$ was deleted. Then place $x$ in that border position and move it to a standard position in $T$ using reverse jeu de taquin. That is, iteratively swap $x$ with the larger of the numbers just above it or just to its left. Reading the above example backwards is an example of reverse jeu de taquin.

A bijective proof of the $S_{n}$ identity $k !=\sum_{\lambda \vdash k}\left(f^{\lambda}\right)^{2}$ was originally found by Robinson [Rob] and later found, independently and in the form we present here, by Schensted [Sch]. Knuth [Kn] analyzed this algorithm and extended it to prove the identity (1.1.a). See [Sta, $\S 7$ Notes] for a nice history of the RSK algorithm. Let $S$ be a tableau of partition shape $\mu$, with $|\mu|<n$, with increasing rows and columns, and with distinct entries from $\{1, \ldots, n\}$. Let $x$ be a positive integer that is not in $S$. The following algorithm inserts $x$ into $S$ producing a standard tableau $T$ of shape $\lambda$ with $\mu \subseteq \lambda,|\lambda / \mu|=1$, whose entries are the union of those from $S$ and $\{x\}$. We denote this process by $x \stackrel{\text { RSK }}{\longrightarrow}$.

1. Let $R$ be the first row of $S$.

2. While $x$ is less than some element in $R$, do

A. Let $y$ be the smallest element of $R$ greater than $x$;

B. Replace $y \in R$ with $x$;

C. Let $x:=y$ and let $R$ be the next row.

3. Place $x$ at the end of $R$ (which is possibly empty).

For example, here is the insertion of 2 into the output of the jeu de taquin example above

\begin{tabular}{|c|c|c|c|c|c|}
\hline 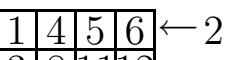 & & \begin{tabular}{|l|l|l|l|}
1 & 2 & 5 & 6 \\
2 & 0 & 1 & 11 \\
\end{tabular} & \begin{tabular}{|l|l|l|l|}
1 & 2 & 5 & 6 \\
2 & & 1 & \\
\end{tabular} & \begin{tabular}{|l|l|l|l|}
1 & 2 & 5 & 6 \\
2 & & 11 & 1 \\
\end{tabular} & \begin{tabular}{|l|l|}
1 & 2 \\
2 & 4
\end{tabular} \\
\hline \begin{tabular}{|l|l|l|}
3 & 8 & 1112 \\
\end{tabular} & $\Rightarrow$ & \begin{tabular}{l|l|l|l|}
3 & 8 & 11 & $-4 \Rightarrow$
\end{tabular} & \begin{tabular}{l|l|l|l|l|}
3 & 4 & 112
\end{tabular}$\Rightarrow$ & \begin{tabular}{|l|l|l|l|l|}
3 & 4 & 112
\end{tabular}$\Rightarrow$ & \begin{tabular}{|l|l|}
3 & 4 \\
\end{tabular} \\
\hline \begin{tabular}{|l|l|}
7 & 10 \\
9 & \\
\end{tabular} & & & \begin{tabular}{l|l}
7 & $10 \longleftarrow 8$ \\
9 & $\leftarrow 8$
\end{tabular} & \begin{tabular}{|l|l|l}
7 & 8 \\
9 & $\leftarrow 10$ \\
\end{tabular} & \begin{tabular}{|l|l|}
7 & 8 \\
9 & 10 \\
\end{tabular} \\
\hline
\end{tabular}

It is possible to invert the process of row insertion using row uninsertion. See [Sag] for details. In the example above, the number 2 and the leftmost tableau are the result of uninserting 10 from the rightmost tableau.

Given $i_{1}, \ldots, i_{k}$, with $1 \leq i_{j} \leq n$, we will produce a pair $\left(T_{\lambda}, P_{\lambda}\right), \lambda \in \Lambda_{n}^{k}$, consisting of a standard tableau $T_{\lambda}$ and a vacillating tableau $P_{\lambda}$. First, initialize the 0 th tableau to 
be the standard tableaux of shape $(n)$, namely,

$$
T^{(0)}=\begin{array}{|l|l|l|l|}
\hline 1 & 2 & \cdots & n \\
\hline
\end{array}
$$

Then, recursively define standard tableaux $T^{\left(j+\frac{1}{2}\right)}$ and $T^{(j+1)}$ by

$$
\begin{aligned}
T^{\left(j+\frac{1}{2}\right)} & =\left(i_{j+1} \stackrel{\mathrm{jdt}}{\longleftrightarrow} T^{(j)}\right), \\
T^{(j+1)} & =\left(i_{j+1} \stackrel{\operatorname{RSK}}{\longrightarrow} T^{\left(j+\frac{1}{2}\right)}\right),
\end{aligned}
$$

Let $\lambda^{(j)} \in \Lambda_{n}^{j}$ be the shape of $T^{(j)}$, and let $\lambda^{\left(j+\frac{1}{2}\right)} \in \Lambda_{n-1}^{j}$ be the shape of $T^{\left(j+\frac{1}{2}\right)}$. Then let

$$
P_{\lambda}=\left(\lambda^{(0)}, \lambda^{\left(\frac{1}{2}\right)}, \lambda^{(1)}, \lambda^{\left(1 \frac{1}{2}\right)}, \ldots, \lambda^{(k)}\right) \quad \text { and } \quad T_{\lambda}=T^{(k)},
$$

so that $P_{\lambda}$ is a vacillating tableau of shape $\lambda=\lambda^{(k)} \in \Lambda_{n}^{k}$, and $T_{\lambda}$ is a standard tableaux of the same shape $\lambda$. We denote this iterative "delete-insert" process that associates the pair $\left(T_{\lambda}, P_{\lambda}\right)$ to the sequence $\left(i_{1}, \ldots, i_{k}\right)$ by

$$
\left(i_{1}, \ldots, i_{k}\right) \stackrel{\mathrm{DI}}{\longrightarrow}\left(T_{\lambda}, P_{\lambda}\right) .
$$

Figure 3 shows the calculations which give

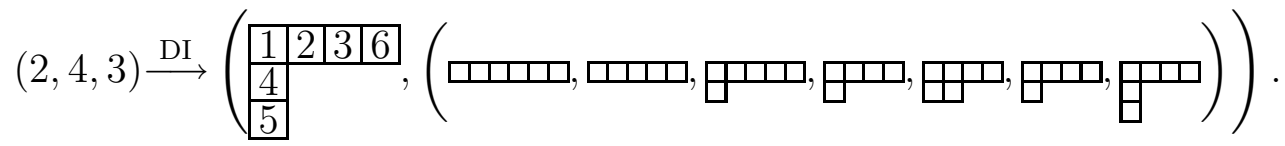

Theorem 3.1. The function $\left(i_{1}, \ldots, i_{k}\right) \stackrel{\mathrm{DI}}{\longrightarrow}\left(T_{\lambda}, P_{\lambda}\right)$ provides a bijection between sequences in $\left\{\left(i_{1}, \ldots, i_{k}\right) \mid 1 \leq i_{j} \leq n\right\}$ and $\bigsqcup_{\lambda \in \Lambda_{n}^{k}} \mathcal{S} \mathcal{Y} \mathcal{T}(\lambda) \times \mathcal{V} \mathcal{T}_{k}(\lambda)$ and thus gives a combinatorial proof of (3.1).

Proof. We prove the theorem by constructing the inverse of $\stackrel{\mathrm{DI}}{\longrightarrow}$.

Let $\lambda^{\left(j+\frac{1}{2}\right)} \subseteq \lambda^{(j+1)}$ with $\lambda^{(j+1)} \in \Lambda_{n}^{(j+1)}$ and $\lambda^{\left(j+\frac{1}{2}\right)} \in \Lambda_{n-1}^{j}$, and let $T^{(j+1)}$ be a standard tableau of shape $\lambda^{(j+1)}$. We can uniquely determine $i_{j+1}$ and a tableau $T^{\left(j+\frac{1}{2}\right)}$ of shape $\lambda^{\left(j+\frac{1}{2}\right)}$ such that $T^{(j+1)}=\left(i_{j+1} \stackrel{\mathrm{RSK}}{\longrightarrow} T^{\left(j+\frac{1}{2}\right)}\right)$. To do this let $b$ be the box in $\lambda^{(j+1)} / \lambda^{\left(j+\frac{1}{2}\right)}$. Then let $i_{j+1}$ and $T^{\left(j+\frac{1}{2}\right)}$ be the result of uninserting the number in box $b$ of $T^{(j+1)}$ (using the fact that RSK insertion is invertible).

Now let $T^{\left(j+\frac{1}{2}\right)}$ be a tableau of shape $\lambda^{\left(j+\frac{1}{2}\right)} \in \Lambda_{n-1}^{(j)}$ with increasing rows and columns and entries $\{1, \ldots, n\} \backslash\left\{i_{j+1}\right\}$ and let $\lambda^{(j)} \subseteq \lambda^{\left(j+\frac{1}{2}\right)}$ with $\lambda^{(j)} \in \Lambda_{n}^{j}$. We can uniquely produce a standard tableau $T^{(j)}$ such that $T^{\left(j+\frac{1}{2}\right)}=\left(i_{j+1} \stackrel{\text { jdt }}{\longleftarrow} T^{(j)}\right)$. To do this, let $b$ be the box in $\lambda^{(j)} / \lambda^{\left(j+\frac{1}{2}\right)}$, put $i_{j+1}$ in position $b$ of $T^{\left(j+\frac{1}{2}\right)}$, and perform the inverse of jeu de taquin to produce $T^{(j)}$. That is, move $i_{j+1}$ into a standard position by iteratively swapping it with the larger of the numbers just above it or just to its left.

Given $\lambda \in \Lambda_{n}^{k}$ and $\left(P_{\lambda}, T_{\lambda}\right) \in \mathcal{S} \mathcal{Y} \mathcal{T}(\lambda) \times \mathcal{V} \mathcal{T}_{k}(\lambda)$, we apply the process above to $\lambda^{\left(k-\frac{1}{2}\right)} \subseteq \lambda^{(k)}$ and $T^{(k)}=T_{\lambda}$ producing $i_{k}$ and $T^{(k-1)}$. Continuing this way, we can produce $i_{k}, i_{k-1}, \ldots, i_{1}$ and $T^{(k)}, T^{(k-1)}, \ldots, T^{(1)}$ such that $\left(i_{1}, \ldots, i_{k}\right) \stackrel{\mathrm{DI}}{\longrightarrow}\left(T_{\lambda}, P_{\lambda}\right)$. 
Figure 3: Delete-Insertion of $(2,4,3)$

\begin{tabular}{|c|c|c|}
\hline$j$ & $i_{j}$ & $T^{(j)}$ \\
\hline 0 & & 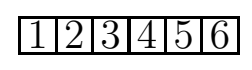 \\
\hline$\frac{1}{2}$ & $2 \stackrel{\text { jdt }}{\longleftarrow}$ & \begin{tabular}{|l|l|l|l|l|}
1 & 3 & 4 & 5 & 6 \\
\end{tabular} \\
\hline 1 & $2 \stackrel{\mathrm{RSK}}{\longrightarrow}$ & \begin{tabular}{|l|l|l|l|}
1 & 2 & 4 & 5 \\
3 & & \\
\end{tabular} \\
\hline $1 \frac{1}{2}$ & $4 \stackrel{\mathrm{jdt}}{\longleftrightarrow}$ & \begin{tabular}{|l|l|l|}
1 & 2 & 5 \\
3 & & \\
\end{tabular} \\
\hline 2 & $4 \stackrel{\mathrm{RSK}}{\longrightarrow}$ & \begin{tabular}{|l|l|l|}
1 & 2 & 4 \\
3 & 5 & \\
\end{tabular} \\
\hline $2 \frac{1}{2}$ & $3 \stackrel{\mathrm{jdt}}{\longleftarrow}$ & \begin{tabular}{|l|l|l|}
1 & $2|4| 6$ \\
5 & \\
\end{tabular} \\
\hline 3 & $3 \stackrel{\mathrm{RSK}}{\longrightarrow}$ & 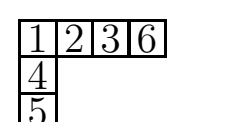 \\
\hline
\end{tabular}

\section{A Bijective Proof of $B(2 k)=\sum_{\lambda \in \Gamma_{k}}\left(m_{k}^{\lambda}\right)^{2}$}

If we view $\mathbb{C} A_{k}(n)$ as a bimodule for $\mathbb{C} A_{k}(n) \otimes \mathbb{C} A_{k}(n)$, with the first tensor factor acting by left multiplication on $\mathbb{C} A_{k}(n)$ and the second tensor factor acting by right multiplication on $\mathbb{C} A_{k}(n)$, then the decomposition into irreducibles for $\mathbb{C} A_{k}(n) \otimes \mathbb{C} A_{k}(n)$ is $\mathbb{C} A_{k}(n) \cong \bigoplus_{\lambda \in \Gamma_{k}} M_{k}^{\lambda} \otimes \bar{M}_{k}^{\lambda}$, where $\bar{M}_{k}^{\lambda}$ is the irreducible right $\mathbb{C} A_{k}(n)$-module indexed by $\lambda$ (so $M_{k}^{\lambda} \cong \bar{M}_{k}^{\lambda}$ ). This is a generalization of the decomposition of the group algebra of a finite group. Comparing dimensions on both sides gives,

$$
B(2 k)=\sum_{\lambda \in \Gamma_{k}}\left(m_{k}^{\lambda}\right)^{2} .
$$

For example using the numbers $m_{3}^{\lambda}$ from the bottom row of Figure 2, we have $B(6)=$ $203=5^{2}+10^{2}+6^{2}+6^{2}+1^{2}+2^{2}+1^{2}$.

To give a combinatorial proof of (4.1), we find a bijection of the form

$$
A_{k} \longleftrightarrow \bigsqcup_{\lambda \in \Gamma_{k}} \mathcal{V} \mathcal{T}_{k}(\lambda) \times \mathcal{V} \mathcal{T}_{k}(\lambda)
$$

by constructing a function that takes a set partition $d \in A_{k}$ and produces a pair $\left(P_{\lambda}, Q_{\lambda}\right)$ of vacillating tableaux. In Section 5 we show that our bijection restricts to work for 
all the diagram subalgebras described in Section 2. In particular, if $d$ corresponds to a permutation in $S_{k}$ then our bijection is the usual RSK algorithm described in Section 3.2.

We will draw diagrams $d \in A_{k}$ using a standard representation as single row with the vertices in order $1, \ldots, 2 k$, where we relabel vertex $j^{\prime}$ with the label $2 k-j+1$. We draw the edges of the standard representation of $d \in A_{k}$ in a specific way: connect vertices $i$ and $j$, with $i \leq j$, if and only if $i$ and $j$ are related in $d$ and there does not exist $k$ related to $i$ and $j$ with $i<k<j$. In this way, each vertex is connected only to its nearest neighbors in its block. For example,
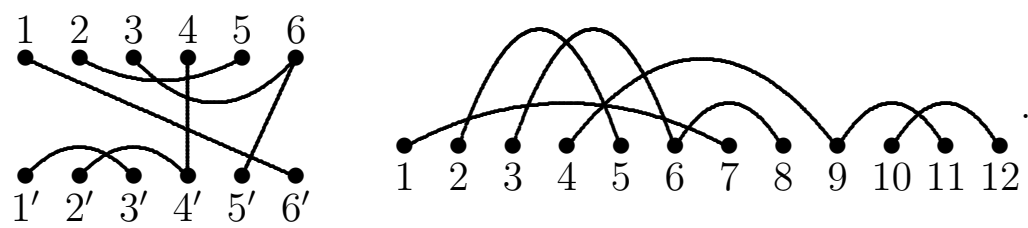

We label each edge $e$ of the diagram $d$ with $2 k+1-\ell$, where $\ell$ is the right vertex of $e$. Define the insertion sequence of a diagram to be the sequence $E=\left(E_{j}\right)$ indexed by $j$ in the sequence $\frac{1}{2}, 1,1 \frac{1}{2}, \ldots, 2 k-1,2 k-\frac{1}{2}, 2 k$, where

$$
\begin{aligned}
E_{j} & = \begin{cases}a, & \text { if vertex } j \text { is the left endpoint of edge } a, \\
\emptyset, & \text { if vertex } j \text { is not a left endpoint }\end{cases} \\
E_{j-\frac{1}{2}} & = \begin{cases}a, & \text { if vertex } j \text { is the right endpoint of edge } a, \\
\emptyset, & \text { if vertex } j \text { is not a right endpoint. }\end{cases}
\end{aligned}
$$

Example 4.1. The edge labeling for the diagram of the set partition $\left\{\left\{1,3,4^{\prime}\right\},\left\{2,1^{\prime}\right\}\right.$, $\left.\left\{4,3^{\prime}, 2^{\prime}\right\}\right\}$ is given by
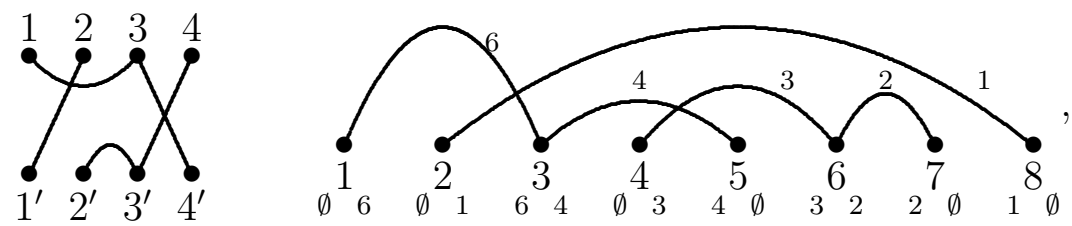

and the insertion sequence is

\begin{tabular}{c|cccccccccccccccc}
\hline$j$ & $\frac{1}{2}$ & 1 & $1 \frac{1}{2}$ & 2 & $2 \frac{1}{2}$ & 3 & $3 \frac{1}{2}$ & 4 & $4 \frac{1}{2}$ & 5 & $5 \frac{1}{2}$ & 6 & $6 \frac{1}{2}$ & 7 & $7 \frac{1}{2}$ & 8 \\
\hline$E_{j}$ & $\emptyset$ & 6 & $\emptyset$ & 1 & 6 & 4 & $\emptyset$ & 3 & 4 & $\emptyset$ & 3 & 2 & 2 & $\emptyset$ & 1 & $\emptyset$ \\
\hline
\end{tabular}

The insertion sequence of a standard diagram completely determines the edges, and thus the connected components, of the diagram, so the following proposition follows immediately.

Proposition 4.2. $d \in A_{k}$ is completely determined by its insertion sequence. 
For $d \in A_{k}$ with insertion sequence $E=\left(E_{j}\right)$, we will produce a pair $\left(P_{\lambda}, Q_{\lambda}\right)$ of vacillating tableaux. Begin with the empty tableaux,

$$
T^{(0)}=\emptyset \text {. }
$$

Then recursively define standard tableaux $T^{\left(j+\frac{1}{2}\right)}$ and $T^{(j+1)}$ by

$$
\begin{aligned}
T^{\left(j+\frac{1}{2}\right)} & = \begin{cases}E_{j+\frac{1}{2}} \stackrel{\mathrm{jdt}}{\longleftrightarrow} T^{(j)}, & \text { if } E_{j+\frac{1}{2}} \neq \emptyset, \\
T^{(j)}, & \text { if } E_{j+\frac{1}{2}}=\emptyset,\end{cases} \\
T^{(j+1)} & = \begin{cases}E_{j+1} \stackrel{\mathrm{RSK}}{\longrightarrow} T^{\left(j+\frac{1}{2}\right)}, & \text { if } E_{j+1} \neq \emptyset, \\
T^{\left(j+\frac{1}{2}\right)}, & \text { if } E_{j+1}=\emptyset,\end{cases}
\end{aligned}
$$

Let $\lambda^{(i)}$ be the shape of $T^{(i)}$, let $\lambda^{\left(i+\frac{1}{2}\right)}$ be the shape of $T^{\left(i+\frac{1}{2}\right)}$, and let $\lambda=\lambda^{(k)}$. Define

$$
\begin{aligned}
Q_{\lambda} & =\left(\emptyset, \lambda^{\left(\frac{1}{2}\right)}, \lambda^{(1)}, \ldots, \lambda^{\left(k-\frac{1}{2}\right)}, \lambda^{(k)}\right), \\
P_{\lambda} & =\left(\lambda^{(2 k)}, \lambda^{\left(2 k-\frac{1}{2}\right)}, \ldots, \lambda^{\left(k+\frac{1}{2}\right)}, \lambda^{(k)}\right) .
\end{aligned}
$$

In this insertion process, every edge of the partition diagram is inserted when we come to its left endpoint and deleted when we come to its right endpoint, so the final shape is $\lambda^{(2 k)}=\emptyset$. This means that $P_{\lambda} \in \mathcal{V} \mathcal{T}(\lambda)$ and $Q_{\lambda} \in \mathcal{V} \mathcal{T}(\lambda)$, so we have associated a pair of vacillating tableaux $\left(P_{\lambda}, Q_{\lambda}\right)$ to a set partition $d \in A_{k}$. We denote this we process by

$$
d \rightarrow\left(P_{\lambda}, Q_{\lambda}\right) .
$$

In Figure 4, we see the insertion of the diagram in Example 4.1. The result of this is to pair the diagram in Example 4.1 with the following pair of vacillating tableaux,

$$
\begin{aligned}
Q_{\lambda} & =(\emptyset, \emptyset, \square, \square, \boxminus, \square, \square, \square, \square), \\
P_{\lambda} & =(\emptyset, \emptyset, \square, \square, \square, \square, \square, \square, \square) .
\end{aligned}
$$

Remark 4.3. We have numbered the edges of the standard diagram of $d$ in increasing order from right to left, so if $E_{j+\frac{1}{2}} \neq \emptyset$, then $E_{j+\frac{1}{2}}$ will be the largest element of $T^{(j)}$. Thus, in $T^{\left(j+\frac{1}{2}\right)}=\left(E_{j+\frac{1}{2}} \stackrel{\mathrm{jdt}}{\longleftarrow} T^{(j)}\right)$ we know that $E_{j+\frac{1}{2}}$ is in a corner box, and jeu de taquin simply deletes that box.

Theorem 4.4. The function $d \rightarrow\left(P_{\lambda}, Q_{\lambda}\right)$ provides a bijection between the set partitions $A_{k}$ and pairs of vacillating tableaux $\bigsqcup_{\lambda \in \Gamma_{k}} \mathcal{V} \mathcal{T}(\lambda) \times \mathcal{V} \mathcal{T}_{k}(\lambda)$ and thus gives a combinatorial proof of (4.1).

Proof. We prove the theorem by constructing the inverse of $d \rightarrow\left(P_{\lambda}, Q_{\lambda}\right)$. First, we use $Q_{\lambda}$ followed by $P_{\lambda}$ in reverse order to construct the sequence $\lambda^{\left(\frac{1}{2}\right)}, \lambda^{(1)}, \ldots, \lambda^{\left(2 k-\frac{1}{2}\right)}, \lambda^{(2 k)}$. We initialize $T^{(2 k)}=\emptyset$. 
Figure 4: Insertion of the Set Partition in Example 4.1.

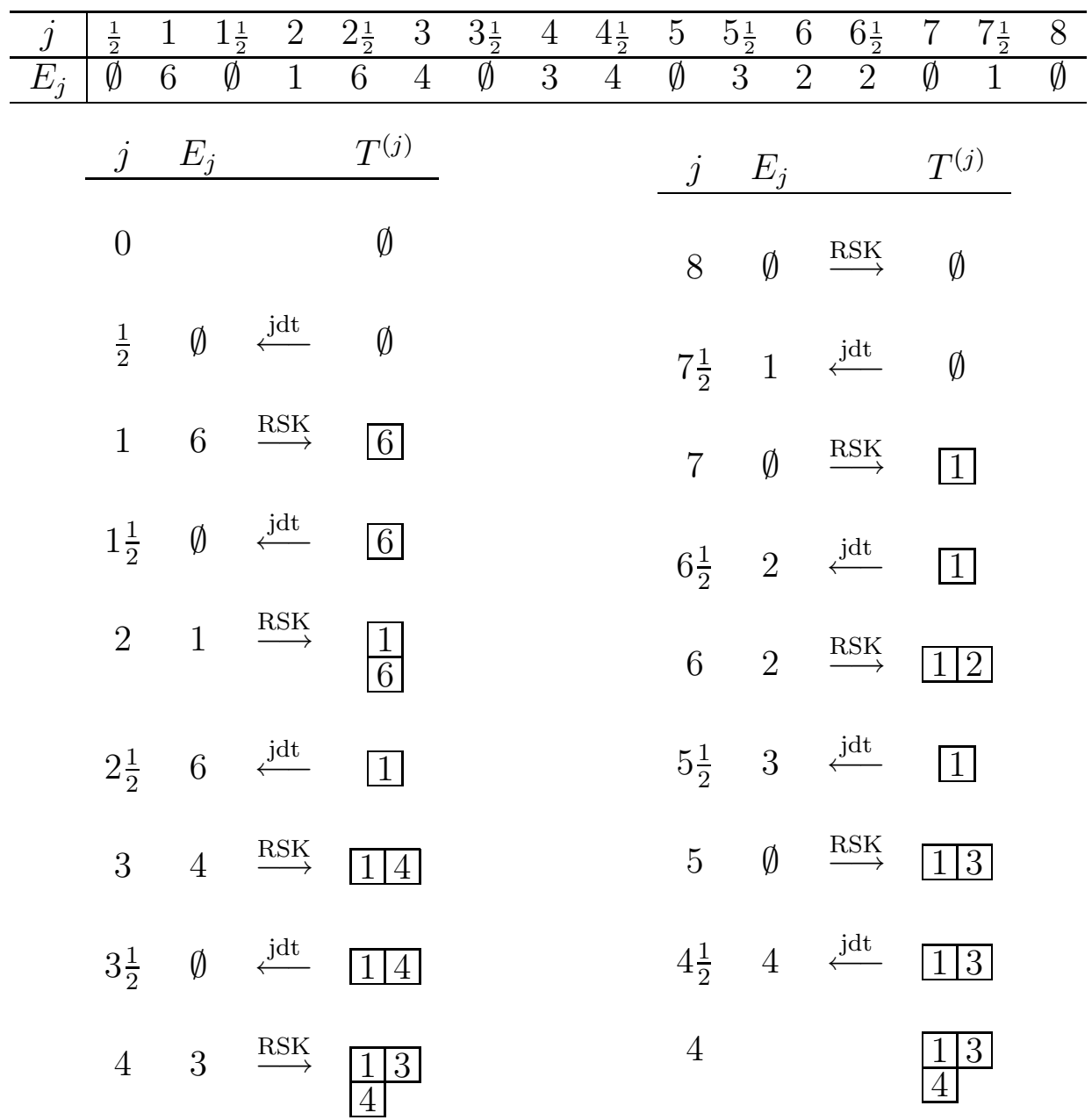

We now show how to construct $T^{\left(i+\frac{1}{2}\right)}$ and $E_{i+1}$ so that $T^{(i+1)}=\left(E_{i+1} \stackrel{\mathrm{RSK}}{\longrightarrow} T^{\left(i+\frac{1}{2}\right)}\right)$. If $\lambda^{\left(i+\frac{1}{2}\right)}=\lambda^{(i+1)}$, then let $T^{\left(i+\frac{1}{2}\right)}=T^{(i+1)}$ and $E_{i+1}=\emptyset$. Otherwise, $\lambda^{(i+1)} / \lambda^{\left(i+\frac{1}{2}\right)}$ is a box $b$, and we use uninsertion on the value in $b$ to produce $T^{\left(i+\frac{1}{2}\right)}$ and $E_{i+1}$ such that $T^{(i+1)}=\left(E_{i+1} \stackrel{\mathrm{RSK}}{\longrightarrow} T^{\left(i+\frac{1}{2}\right)}\right)$. Since we uninserted the value in position $b$, we know that $T^{\left(i+\frac{1}{2}\right)}$ has shape $\lambda^{\left(i+\frac{1}{2}\right)}$.

We then show how to construct $T^{(i)}$ and $E_{i+\frac{1}{2}}$ so that $T^{\left(i+\frac{1}{2}\right)}=\left(E_{i+\frac{1}{2}} \stackrel{\mathrm{jdt}}{\longleftarrow} T^{(i)}\right)$. If $\lambda^{(i)}=\lambda^{\left(i+\frac{1}{2}\right)}$, then let $T^{(i)}=T^{\left(i+\frac{1}{2}\right)}$ and $E_{i+\frac{1}{2}}=\emptyset$. Otherwise, $\lambda^{(i)} / \lambda^{\left(i+\frac{1}{2}\right)}$ is a box $b$. Let $T^{(i)}$ be the tableau of shape $\lambda^{(i)}$ with the same entries as $T^{(i+1)}$ and having the entry $2 k-i$ in box $b$. Let $E_{i+\frac{1}{2}}=2 k-i$. At any given step $i$ (as we work our way down from step $2 k$ to step $\left.\frac{1}{2}\right), 2 k-i$ is the largest value added to the tableau thus far, so $T^{(i)}$ is standard. Furthermore, $T^{\left(i+\frac{1}{2}\right)}=\left(E_{i+\frac{1}{2}} \stackrel{\mathrm{jdt}}{\longleftarrow} T^{(i)}\right)$, since $E_{i+\frac{1}{2}}=2 k-i$ is already in a corner and thus 
jeu de taquin simply deletes it.

This iterative process will produce $E_{2 k}, E_{2 k-1}, \ldots, E_{\frac{1}{2}}$ which completely determines the set partition $d$. By the way we have constructed $d$, we have $d \rightarrow\left(P_{\lambda}, Q_{\lambda}\right)$.

\section{Properties of the Insertion Algorithm}

In this section we investigate a number of the nice properties of the insertion algorithm from Section 4. First, we note that our algorithm restricts to the insertion algorithms for the subalgebras discussed in Section 1:

Remark 5.1. (a) If $d \in S_{k}$ is a permutation diagram, then the standard representation of $d$ pairs vertices $1, \ldots k$ with vertices $k+1, \ldots, 2 k$. Our algorithm inserts on steps $j$, with $1 \leq j \leq k$, and deletes on steps $j+\frac{1}{2}$, with $k \leq j \leq 2 k-1$. The algorithm does nothing on all other steps. If we ignore these "do-nothing" steps, then the algorithm becomes the usual RSK insertion and $P_{\lambda}$ and $Q_{\lambda}$ are the insertion and recording tableaux, respectively.

(b) If $d \in B_{k}$ is a Brauer diagram, then each vertex in the standard representation of $d$ is incident to exactly one edge, so exactly one of $E_{\left(j-\frac{1}{2}\right)}$ or $E_{j}$ will be nonempty for each $1 \leq j \leq 2 k$. If we ignore the steps where we do nothing, then for each vertex we either insert or delete the edge number associated to that vertex. This is precisely the insertion scheme of Sundaram [Sun] (see also [Ter]). This algorithm produces "oscillating tableaux," which either increase or decrease by one box on each step. If we insert all the Brauer diagrams, we produce the Bratteli diagram for $\mathbb{C} B_{k}(n)$ (see for example [HR1]).

(c) If $d \in R_{k}$ is a rook monoid diagram, then our algorithm has us insert or do nothing on steps $0<j \leq k$ and delete or do nothing on steps $j+\frac{1}{2}$ with $k \leq j \leq 2 k-1$. If we insert all rook monoid diagrams, we obtain the Bratteli diagram for $\mathbb{C} R_{k}$ (see $[\mathrm{Ha}])$.

(d) If $d \in A_{k-\frac{1}{2}}$ then the standard representation of $d$ has an edge, labeled $k$, connecting vertices $k$ and $k+1$. At step $k$ in the insertion process, we will insert this edge into $T^{\left(k-\frac{1}{2}\right)}$. The label $k$ is larger than all the entries in $T^{\left(k-\frac{1}{2}\right)}$, since the entries that are in $T^{\left(k-\frac{1}{2}\right)}$ correspond to edges whose right endpoint is larger than $k+1$. Thus we insert this entry at the end of the first row and in the next step, $k+\frac{1}{2}$, we immediately delete it, so that $\lambda^{\left(k-\frac{1}{2}\right)}=\lambda^{\left(k+\frac{1}{2}\right)}$. If we remove $\lambda^{(k)}$ from our pair of paths, we obtain a pair of vacillating tableaux $\left(P_{\lambda^{\left(k-\frac{1}{2}\right)}}, Q_{\lambda^{\left(k-\frac{1}{2}\right)}}\right)$ of length $2 k-1$, and we get a combinatorial formula for the following dimension identity for $\mathbb{C} A_{k-\frac{1}{2}}(n)$ involving the odd Bell numbers,

$$
B(2 k-1)=\sum_{\lambda \in \Gamma_{k-\frac{1}{2}}}\left(m_{k-\frac{1}{2}}^{\lambda}\right)^{2} .
$$


Proposition 5.2. If $d \in A_{k}$ such that $d \rightarrow\left(P_{\lambda}, Q_{\lambda}\right)$, then $|\lambda|=\operatorname{pn}(d)$, where $\operatorname{pn}(d)$ is the propagating number defined in (2.3).

Proof. If we draw a vertical line between vertex $k$ and $k+1$ in the standard representation of $d$, then $\operatorname{pn}(d)$ is the number of edges that cross this line. The labels of these edges are exactly the numbers which have been inserted but not deleted at step $k$ of our algorithm. Thus the tableau $T^{(k)}$ has exactly these numbers in it and so $\lambda^{(k)}$ consists of pn $(d)$ boxes.

Recall that $I_{t}$ is spanned by the diagrams whose propagating number satisfies $\mathrm{pn}(d) \leq$ t. The chain of ideals $\mathbb{C} I_{0}(n) \subseteq \mathbb{C} I_{1}(n) \subseteq \cdots \subseteq \mathbb{C} I_{k}(n)=\mathbb{C} A_{k}(n)$ is a key feature of the "basic construction" of $\mathbb{C} A_{k}(n)$ (see [HR2, Mar3]). The irreducible components of $\mathbb{C} I_{t}(n) /\left(\mathbb{C} I_{t-1}(n)\right)$ are those indexed by $\lambda \in \Gamma_{k}$ with $|\lambda|=t$. The previous proposition tells us that our insertion algorithm respects this basic construction and gives a combinatorial proof of the following dimension identity for $\mathbb{C} I_{t}(n) /\left(\mathbb{C} I_{t-1}(n)\right)$,

$$
\operatorname{Card}\left(\left\{d \in A_{k} \mid \operatorname{pn}(d)=t\right\}\right)=\sum_{\substack{\lambda \in \Gamma_{k} \\|\lambda|=t}}\left(m_{k}^{\lambda}\right)^{2} .
$$

Proposition 5.3. Let $d \rightarrow(P, Q)=\left(\lambda^{(0)}, \ldots, \lambda^{(2 k)}\right)$. Then $d$ is planar if and only if the length of every partition in the sequence satisfies $\ell\left(\lambda^{(i)}\right) \leq 1$.

Proof. This is a special case of the crossing and nesting theorem in [CDDSY]. If $d$ is planar, then the standard representation of $d$ is also planar. For example,
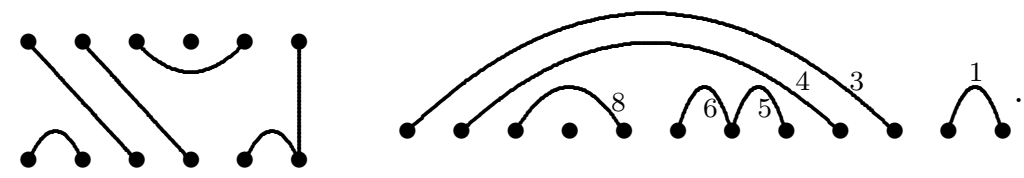

Let $a<b$ be the labels of two edges in a planar diagram $d$ and suppose that $b$ is inserted before $a$ in the insertion sequence of $d$. Since $a<b$ the right vertex of $b$ is left of the right vertex of $a$. Since $b$ is inserted before $a$, the left vertex of $b$ is left of the left vertex of $a$. Since $d$ is planar, the right vertex of $b$ must then be left of the left vertex of $a$. Thus, $b$ is inserted and deleted before $a$ is inserted. This means that when we are inserting an edge label $a$ into $T^{(i)}$, we know that $a$ is larger than all the entries of $T^{(i)}$, and so $a$ placed at the end of the first row. Thus at each step of the insertion, we only ever obtain the empty tableau or a tableau consisting of 1 row. Conversely, to obtain a partition that has more than one column, there must be a pair $a<b$ such that $a$ is inserted after $b$ is inserted but before $b$ is deleted. This is necessary for $a$ to bump $b$ into the next row. By the reasoning above, this forces an edge crossing in the partition diagram.

Remark 5.4. (a) The planar partition diagrams $d \in P_{k}$ are exactly paired with the paths $(P, Q)$ whose vertices contain only length 0 or 1 partitions. The planar partition algebra $\mathbb{C} P_{k}(n)$ is isomorphic to the Temperley-Lieb algebra $\mathbb{C} T_{2 k}(n), k \in \frac{1}{2} \mathbb{Z}_{>0}$, 
(see [Jo] or [HR2]) and has the Bratteli diagram shown in Figure 5. Our algorithm gives a combinatorial proof of the following dimension identity for $\mathbb{C} T_{2 k}(n)$,

$$
C(2 k)=\sum_{\substack{\lambda \in \Lambda_{k} \\
\ell(\lambda) \leq 1}}\left(\left(\begin{array}{c}
2 k \\
\lfloor k\rfloor-|\lambda|
\end{array}\right)-\left(\begin{array}{c}
2 k \\
\lfloor k\rfloor-|\lambda|-1
\end{array}\right)\right)^{2}, \quad k \in \frac{1}{2} \mathbb{Z}_{>0}
$$

where \lfloor\rfloor is the floor function and $\left(\begin{array}{c}2 k \\ \lfloor k-|\lambda|\end{array}\right)-\left(\begin{array}{c}2 k \\ \lfloor k\rfloor-|\lambda|-1\end{array}\right)$ is the dimension of the irreducible $\mathbb{C} T_{2 k}(n)$-module labeled by $\lambda$ (this is also the number of paths to $\lambda$ in the Bratteli diagram of $\left.\mathbb{C} T_{2 k}(n)\right)$.

(b) The planar Brauer diagrams $T_{k}$ span another copy of the Temperley-Lieb algebra $\mathbb{C} T_{k}(n)$ inside $\mathbb{C} A_{k}(n)$, and our insertion algorithm when applied to these diagrams gives a different bijective proof of the identity in (a).

(c) In the insertion for planar rook monoid diagrams in $P R_{k}$, we insert or do nothing for steps $j$ with $0<j \leq k$, we delete or do nothing for steps $j+\frac{1}{2}$ with $k \leq j<2 k$, and we do nothing on all other steps. Thus, the Bratteli diagram, which is shown in Figure 5, has levels $j$ and $j+\frac{1}{2}$ merged and is isomorphic to Pascal's triangle. The irreducible representation associated to the partition of shape $\lambda=(\ell)$ has dimension $\left(\begin{array}{l}k \\ \ell\end{array}\right)$ (see $[\mathrm{HH}]$ for the representation theory of $\left.P R_{k}\right)$. Our algorithm gives an RSK insertion proof of following the well-known binomial identity,

$$
\left(\begin{array}{c}
2 k \\
k
\end{array}\right)=\sum_{\ell=0}^{k}\left(\begin{array}{l}
k \\
\ell
\end{array}\right)^{2}
$$

Finally, we show that the algorithm has the symmetry property of the usual RSK algorithm for the symmetric group. Namely, if $\pi \rightarrow(P, Q)$ for $\pi \in S_{k}$, then $\pi^{-1} \rightarrow(Q, P)$. Using diagrams, the inverse of $\pi$ is simply the diagram of $\pi$ reflected over its horizontal axis. We define the flip of any partition diagram to be this reflection, so for example,

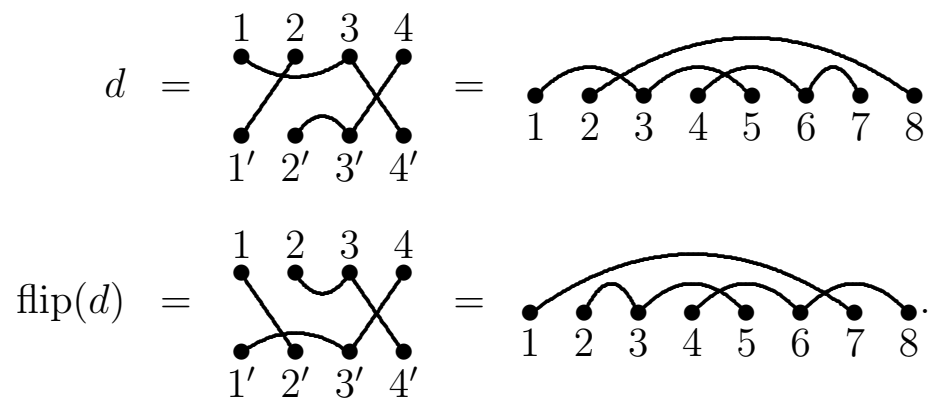

Notice that in the standard representation of $d$, a flip corresponds to a reflection over the vertical line between vertices $k$ and $k+1$. Our goal is to show that if $d \rightarrow(P, Q)$, then $\operatorname{flip}(d) \rightarrow(Q, P)$.

Given a partition $d \in A_{k}$, construct a triangular grid in the integer lattice $\mathbb{Z} \times \mathbb{Z}$ that contains the points in the triangle whose vertices are $(0,0),(2 k, 0)$ and $(0,2 k)$. Number 
Figure 5: Bratteli Diagrams for the Planar Partition Algebra $\mathbb{C} P_{k}(n)$ and the Planar Rook Monoid $P R_{k}$

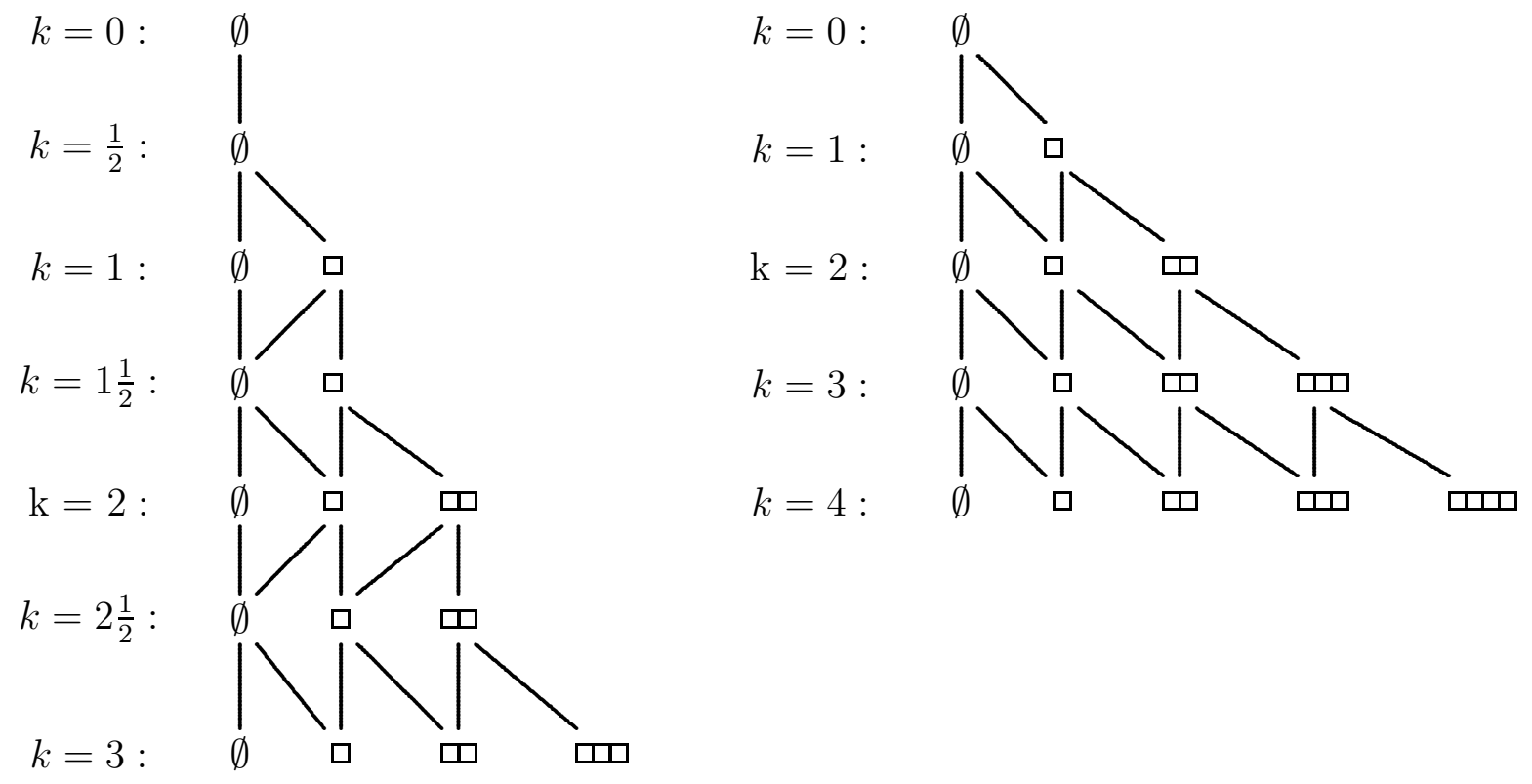

the columns $1, \ldots, 2 k$ from left to right and the rows $1, \ldots, 2 k$ from bottom to top. Place an $\mathbf{X}$ in the box in column $i$ and row $j$ if and only if, in the one-row diagram of $d$, vertex $i$ is the left endpoint of edge $j$. We then label the vertices of the diagram on the bottom row and left column with the empty partition $\emptyset$. For example, in our diagram $d$ from Example 4.1 we have
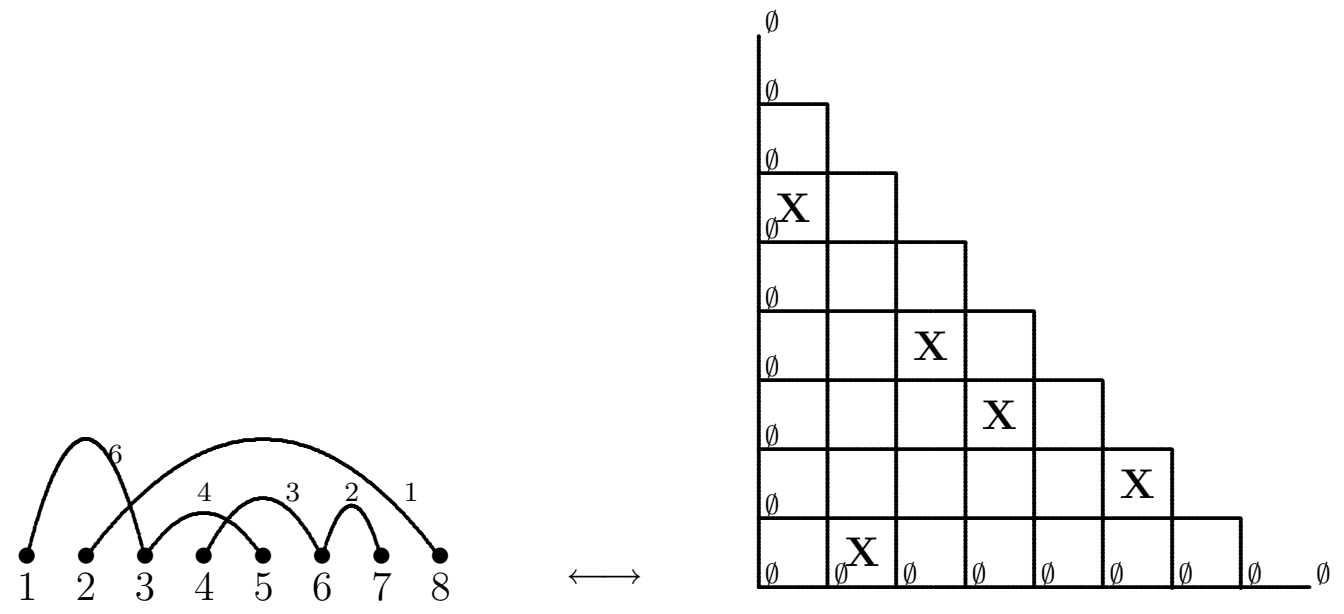

Note that the triangular array completely determines the partition diagram and vice versa.

Now we inductively label the remaining vertices using the local rules of S. Fomin (see [Ry1, Ry2] or [Sta, 7.13] and the references there). If a box is labeled with $\mu, \nu$, and $\lambda$ as 
shown below. Then we add the label $\rho$ according the rules that follow:

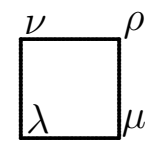

(L1) If $\mu \neq \nu$, let $\rho=\mu \cup \nu$, i.e., $\rho_{i}=\max \left(\mu_{i}, \nu_{i}\right)$.

(L2) If $\mu=\nu, \lambda \subset \mu$, and $\lambda \neq \mu$, then this will automatically imply that $\mu$ can be obtained from $\lambda$ by adding a box to $\lambda_{i}$. Let $\rho$ be obtained from $\mu$ by adding a box to $\mu_{i+1}$.

(L3) If $\mu=\nu=\lambda$, then if the square does not contain an $\mathbf{X}$, let $\rho=\lambda$, and if the square does contain an $\mathbf{X}$, let $\rho$ be obtained from $\lambda$ by adding 1 to $\lambda_{1}$.

Using these rules, we can uniquely label every corner, one sep at a time. The resulting diagram is called the growth diagram $G_{d}$ for $d$. The diagram corresponding to the above example is

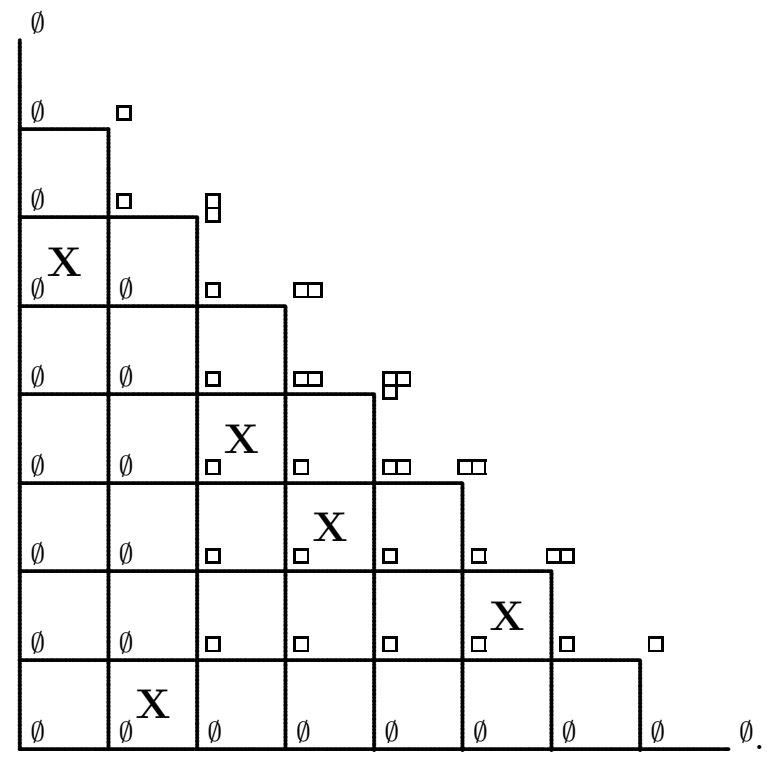

Let $P_{d}$ denote the chain of partitions that follow the staircase path on the diagonal of $G_{d}$ from $(0,2 k)$ to $(k, k)$ and let $Q_{d}$ denote the chain of partitions that follow the staircase path on the diagonal of $G_{d}$ from $(2 k, 0)$ to $(k, k)$. The pair $\left(P_{d}, Q_{d}\right)$ represents a pair of vacillating tableaux whose shape is the partition at $(k, k)$. The staircase path in our growth diagram above is the same as the path we get from insertion in Figure 4.

Theorem 5.5. Let $d \in A_{k}$ with $d \rightarrow(P, Q)$. Then $P_{d}=P$ and $Q_{d}=Q$.

Proof. Turn the diagram $d \in A_{k}$ into a diagram $d^{\prime}$ on $4 k$ vertices by splitting each vertex $i$ into two vertices labeled by $i-\frac{1}{2}$ and $i$. If there is an edge from vertex $j$ to vertex $i$ in $d$, with $j<i$, let $j$ be adjacent to $i-\frac{1}{2}$ in $d^{\prime}$. If there is an edge from vertex $j$ to vertex $i$ 
in $d$, with $j>i$, let $i$ be adjacent to $j-\frac{1}{2}$ in $d^{\prime}$. Thus, in our example we have,
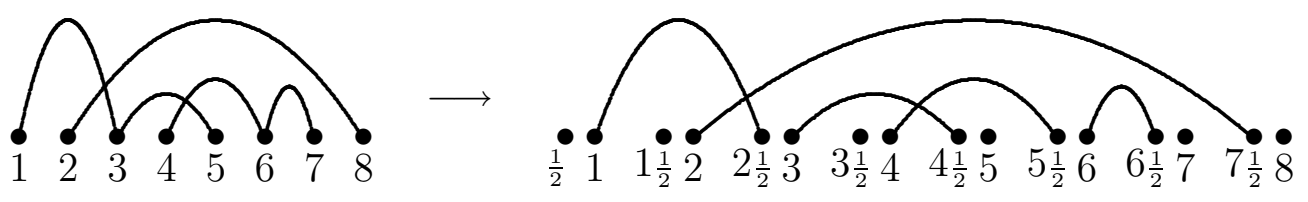

In this way we turn each diagram in $A_{k}$ into a partial matching in $A_{2 k}$. In his dissertation, Roby [Ry1] shows that the up-down tableaux coming from growth diagrams are equivalent to those from RSK insertion of Brauer diagrams (matchings). Dulucq and Sagan [DS] extend this insertion to work for partial matchings (and other generalizations for skew oscillating tableaux) and Roby [Ry2] shows that the growth diagrams also work for the Duluc-Sagan insertion of partial matchings. Furthermore, the insertion sequences that come from our diagram $d$ are the same as the insertion sequences that come from $d^{\prime}$ using the methods of [Sun, DS].

A key advantage of the use of growth diagrams is that the symmetry of the algorithm is nearly obvious. We have that $i$ is the left endpoint of the edge labeled $j$ in $d$ if and only if $j$ is the left endpoint of the edge labeled $i$ in flip $(d)$. Thus the growth diagram of $G_{d}$ is the reflection over the line $y=x$ of the growth diagram of $G_{\text {flip }(d)}$, and so $P_{d}=Q_{\text {flip }(d)}$ and $Q_{d}=P_{\text {flip }(d)}$. Combining this point with the Theorem 5.5 gives

Corollary 5.6. If $d \rightarrow(P, Q)$ then $\operatorname{flip}(d) \rightarrow(Q, P)$.

We say that $d \in A_{k}$ is symmetric if $d=f l i p(d)$. The following is an example of a symmetric diagram in $A_{6}$,
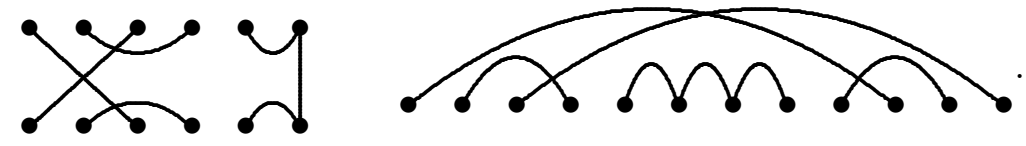

In $S_{k}$ the symmetric diagrams are involutions.

Corollary 5.7. A diagram $d \in A_{k}$ is symmetric if and only if $d \rightarrow(P, P)$.

Proof. If $d$ is symmetric, then by Corollary 5.6 we must have $P=Q$. To prove the converse, let $P=Q$ and place these vacillating tableaux on the staircase border of a growth diagram. The local rules above are invertible: given $\mu, \nu$ and $\rho$ one can the rules backwards to uniquely find $\lambda$ and determine whether there is an $\mathbf{X}$ in the box. Thus, the interior of the growth diagram is uniquely determined. By the symmetry of having $P=Q$ along the staircase, the growth diagram must have a symmetric interior and a symmetric placement of the $\mathrm{X}_{\mathrm{s}}$. This forces $d$ to be symmetric.

This corollary tells us that the number of symmetric diagrams in $A_{k}$ is equal to the number of vacillating tableaux of length $2 k$, or the number of paths to level $k$ in the Bratteli diagram of $\mathbb{C} A_{k}(n)$. Thus,

$$
\operatorname{Card}\left(\left\{d \in A_{k} \mid d \text { is symmetric }\right\}\right)=\sum_{\lambda \in \Lambda_{k}} m_{k}^{\lambda} .
$$


Furthermore, since our insertion restricts to the diagram subalgebras, (5.5) is true if we replace $A_{k}$ with any of the monoids $S_{k}, B_{k}, T_{k}, I_{t}, R_{k}, P R_{k}$ and replace $m_{k}^{\lambda}$ with the dimension of the appropriate irreducible representation. In the case of the symmetric group, (5.5) reduces to the fact that the number of involutions in $S_{k}$ equals $\sum_{\lambda \vdash k} f^{\lambda}$.

\section{References}

[Be] A. Berele, A Schensted-Type Correspondence for the Symplectic Group, J. Combin. Theory Ser. A 43 (1986), no. 2, 320-328.

[Br] R. Brauer, On algebras which are connected with the semisimple continuous groups, Ann. Math. 38 (1937), 854-872.

[CDDSY] W. Chen, E.. Deng, R. Du, R. Stanley, and C. Yan, Crossings and nestings of matchings and partitions, preprint 2005.

[DS] S. Dulucq and B. Sagan, La correspondance de Robinson-Schensted pour les tableaux oscillants gauches, Discrete Math. 139 (1995), 129-142.

[Ha] T. Halverson, Representations of the q-rook monoid, J. Algebra 273 (2004), 227251.

[HH] T. Halverson and K. Herbig, The planar rook monoid, preprint 2005.

[HR1] T. Halverson and A. Ram, Characters of algebras containing a Jones basic construction: the Temperley-Lieb, Okada, Brauer, and Birman-Wenzl algebras. Adv. Math., 116 (1995), 263-321.

[HR2] T. Halverson and A. Ram, Partition Algebras, European J. Combinatorics, 26 (2005), 869-921.

[Jo] V. F. R. Jones, The Potts model and the symmetric group, in: Subfactors: Proceedings of the Taniguchi Symposium on Operator Algebras (Kyuzeso, 1993), World Sci. Publishing, River Edge, NJ, 1994, 259-267.

[Kn] D. E. Knuth, Permutations, matrices, and generalized Young tableaux, Pacific J. Math. 34 (1970), 709-727.

[Mar1] P. Martin, Temperley-Lieb algebras for nonplanar statistical mechanics - the partition algebra construction, J. Knot Theory Ramifications 3 (1994), 51-82.

[Mar2] P. Martin, The structure of the partition algebras, J. Algebra 183 (1996), no. 2, 319-358.

[Mar3] P. Martin, The partition algebra and the Potts model transfer matrix spectrum in high dimensions, J. Phys. A: Math. Gen., 33 (2000), 3669-3695.

[MR] P. Martin and G. Rollet, The Potts model representation and a RobinsonSchensted correspondence for the partition algebra, Compositio Math. 112 (1998), no. 2, 237-254.

[Rob] G. de B. Robinson, On representations of the symmetric group, Amer. J. Math. 60, (1938) 745-760. 
[Ry1] T. Roby, Applications and Extensions of Fomin's Generalization of the RobinsonSchensted Correspondence to Differential Posets, Ph. D. Thesis, M.I.T. 1991.

[Ry2] T. Roby, The connection between the Robinson-Schensted correspondence for skew oscillating tableaux and graded graphs. Discrete Math, 139 (1995), 481485 .

[Sag] B. Sagan, The Symmetric Group, Representations, Combinatorial Algorithms, and Symmetric Functions, Second edition, Springer-Verlag, New York, 2001.

[Sch] C. Schensted, Longest increasing and decreasing subsequences, Canad. J. Math. 13 (1961), 179-191.

[Scü] M. Schützenberger, La correspondence de Robinson, in Combinatoire et Représentation du Groupe Symétrique, D. Foata ed., Lecture Notes in Math. Vol. 579, Springer-Verlag, New York, 1977, 59-135.

[Sta] R. Stanley, Enumerative Combinatorics, Vol. 2, Cambridge University Press, Cambridge, 1999.

[Sun] S. Sundaram, On the combinatorics of representations of $\operatorname{Sp}(2 n \mathbb{C}), \mathrm{Ph}$. D. Thesis, M.I T. 1986.

[Ter] I. Terada, Brauer diagrams, updown tableaux and nilpotent matrices, J. Algebraic Combin., 14 (2001), no. 3, 229-267. 